JOURNAL OF THE

AMERICAN MATHEMATICAL SOCIETY

Volume 10, Number 4, October 1997, Pages 797-833

S 0894-0347(97)00242-7

\title{
RESIDUE FORMULAE, VECTOR PARTITION FUNCTIONS AND LATTICE POINTS IN RATIONAL POLYTOPES
}

\author{
MICHEL BRION AND MICHÈLE VERGNE
}

\section{INTRODUCTION AND STATEMENT OF THE MAIN RESULTS}

The main objects of the present article are vector partition functions, defined as follows. Let $E$ be a real vector space of finite dimension $d$, endowed with a lattice $\Lambda$. Let $\Delta=\left(\alpha_{1}, \ldots, \alpha_{N}\right)$ be a sequence of elements of $\Lambda$, all lying in an open half-space. Then, for $\lambda \in \Lambda$, the equation

$$
\sum_{k=1}^{N} x_{k} \alpha_{k}=\lambda
$$

has a finite number of solutions in non-negative integers $x_{1}, \ldots, x_{N}$. Denote this number by $\mathcal{P}_{\Delta}(\lambda)$, and call $\mathcal{P}_{\Delta}$ the vector partition function associated to $\Delta$.

We begin by discussing our main results (announced in [5] and [6]), their motivations, and their relation to earlier work, in an informal way; precise statements will be given at the end of this introduction. Clearly, the function $\mathcal{P}_{\Delta}$ vanishes outside the closed convex cone $C(\Delta) \subset E$ generated by $\Delta$. We define a subdivision of this cone into closed polyhedral cones. The interiors of maximal cones of this subdivision are called chambers. We obtain a closed formula for restriction of $\mathcal{P}_{\Delta}$ to the closure of each chamber, which displays the periodic polynomial behaviour of this function and its remarkable "continuity" properties under changing of chamber; this refines the result of [20]. More generally, we determine the function

$$
\mathcal{P}_{\Delta}(y, \lambda)=\sum_{x_{1} \alpha_{1}+\cdots+x_{N} \alpha_{N}=\lambda} e^{-x_{1} y_{1}-\cdots-x_{N} y_{N}}
$$

on the closure of each chamber, where $y=\left(y_{1}, \ldots, y_{N}\right)$ is in $\mathbb{R}^{N}$, and where $x_{1}, \ldots, x_{N}$ in the summation are non-negative integers.

Set

$$
V:=\left\{\left(x_{1}, \ldots, x_{N}\right) \in \mathbb{R}^{N} \mid \sum_{k=1}^{N} x_{k} \alpha_{k}=0\right\}
$$

and denote by $n$ the dimension of $V$. For $h \in E$, set

$$
V(h):=\left\{\left(x_{1}, \ldots, x_{N}\right) \in \mathbb{R}^{N} \mid \sum_{k=1}^{N} x_{k} \alpha_{k}=h\right\} \text { and } P_{\Delta}(h):=V(h) \cap\left(\mathbb{R}_{\geq 0}\right)^{N} .
$$

Received by the editors December 30, 1996 and, in revised form, March 28, 1997.

1991 Mathematics Subject Classification. Primary 11P21, 52B20.

Key words and phrases. Vector partition functions, rational convex polytopes. 
Then each $V(h)$ is an affine space with direction $V$, and $P_{\Delta}(h)$ is a convex polytope in $V(h)$. Clearly, $P_{\Delta}(h)$ is not empty if and only if $h \in C(\Delta)$. The family $\left(P_{\Delta}(h)\right)_{h \in C(\Delta)}$ is called the family of partition polytopes defined by $\Delta$. When $h$ is in a chamber, the polytope $P_{\Delta}(h)$ is $n$-dimensional and simple, that is, there are only $n$ edges through each vertex. However, when $h$ moves to the boundary of a chamber, some vertices tend to a single vertex, and $P_{\Delta}(h)$ degenerates.

Observe that

$$
\mathcal{P}_{\Delta}(y, \lambda)=\sum_{\left(x_{1}, \ldots, x_{N}\right) \in P_{\Delta}(\lambda) \cap \mathbb{Z}^{N}} e^{-x_{1} y_{1}-\cdots-x_{N} y_{N}} .
$$

We define a continuous version of the function $\lambda \in C(\Delta) \cap \Lambda \rightarrow \mathcal{P}_{\Delta}(y, \lambda)$ as follows. We normalize the Lebesgue measure on $E$ so that the volume of $E / \Lambda$ is 1 , and we consider the standard Lebesgue measure on $\mathbb{R}^{N}$. These choices determine a Lebesgue measure $d s$ on each $V(h)$. For any linear form $y$ on $\mathbb{R}^{N}$, we set

$$
\mathcal{V}_{\Delta}(y, h):=\int_{P_{\Delta}(h)} e^{-\langle y, s\rangle} d s .
$$

In particular, $\mathcal{V}_{\Delta}(0, h):=\mathcal{V}_{\Delta}(h)$ is the volume of $P_{\Delta}(h)$.

We obtain closed formulae for $\mathcal{P}_{\Delta}(y, \lambda)$ and $\mathcal{V}_{\Delta}(y, h)$ for $\lambda$ and $h$ in the closure of a chamber. We deduce an expression of $\mathcal{P}_{\Delta}(y, \lambda)$ in terms of values at $\lambda$ of the function $h \mapsto \mathcal{V}_{\Delta}(y, h)$ and of its partial derivatives, as an Euler-MacLaurin formula for vector partition functions. Such a formula has also been obtained by Guillemin, see [12].

Consider now a rational convex polytope $P$ in an $n$-dimensional real vector space $V$ endowed with a lattice. Then $P$ can be identified in a canonical way with a polytope $P_{\Delta}(\lambda)$, so that the family $\left(P_{\Delta}(h)\right)$ is obtained from $P$ by independent parallel motions of its facets; see 4.1 below. So our Euler-MacLaurin formula for vector partition functions translates into a universal formula for the number of lattice points (or more generally, for the sum of values of a polynomial function at all lattice points) in a family of rational polytopes with parallel faces. Such a formula was first obtained by Khovanskii and Pukhlikov [17] for certain simple lattice polytopes, and then extended to other classes of lattice polytopes in [15], [7], [3], [11] and [8]. Our formula will be stated at the end of this introduction; it can be seen as a generalization of a result announced in [8].

Back to partition functions, let $z$ be a linear form on $E$ which takes positive values on $\alpha_{1}, \ldots, \alpha_{N}$. Consider the generating function

$$
\sum_{\lambda \in C(\Delta) \cap \Lambda} \mathcal{P}_{\Delta}(y, \lambda) e^{-\langle z, \lambda\rangle}=\prod_{k=1}^{N} \frac{1}{1-e^{-y_{k}-\left\langle z, \alpha_{k}\right\rangle}} .
$$

We obtain a residue formula for this function of $z$, i.e., we decompose it as a sum of "simple terms", each of which is expressed in a set of $d=\operatorname{dim}(E)$ linearly independent variables $\left\langle z, \alpha_{j}\right\rangle$, multiplied by a residue which depends only on $y_{1}, \ldots, y_{N}$. So it is easy to expand each simple term into a power series in $z$; this leads to our formula for $\mathcal{P}_{\Delta}$.

Similarly, we compute $\mathcal{V}_{\Delta}(y, h)$ by considering

$$
\int_{C(\Delta)} \mathcal{V}_{\Delta}(y, h) e^{-\langle z, h\rangle} d h=\prod_{k=1}^{N} \frac{1}{y_{k}+\left\langle z, \alpha_{k}\right\rangle}
$$


and decomposing the latter into "simple fractions" in $z$, multiplied by the corresponding residue in $y$.

We deduce both residue formulae from a geometric result: a decomposition of the standard cone $\left(\mathbb{R}_{\geq 0}\right)^{N}$ into "simple cones" for which the intersection with each $V(h)$ is easily described. This decomposition of the "generating cone" $\left(\mathbb{R}_{\geq 0}\right)^{N}$ leads to a simultaneous decomposition for the family of partition polytopes $P_{\Delta}(h)$, regardless of the way they degenerate when $h$ moves to the boundary of a chamber.

Our detour via convex geometry could be avoided, but it provides a conceptual explanation for some rather complicated formulae. In fact, we have tried to give an elementary and self-contained exposition of our results in their various aspects: convex geometric, algebraic (formal series and their sums) and analytic (Fourier and Laplace transforms), at the risk of being heavy and repetitive.

Although our methods are very direct, the Atiyah-Bott fixed point formula, together with the Kawasaki-Riemann-Roch formula for the index, are underlying our explicit formulae. In the case where all vertices of $P_{\Delta}(\lambda)$ are integral, there is an $n$-dimensional projective algebraic variety $M(\lambda)$ with an effective action of an $n$-dimensional algebraic torus, such that the polytope $P_{\Delta}(\lambda)$ is the image of $M(\lambda)$ under the moment map, the vertices of $P_{\Delta}(\lambda)$ being the images of fixed points. Furthermore, $\mathcal{P}_{\Delta}(y, \lambda)$ is the character of the space of holomorphic sections of the line bundle over $M(\lambda)$, and the higher cohomology groups vanish. So $\mathcal{P}_{\Delta}(y, \lambda)$ is the equivariant index, and, at least for regular $\lambda$, our formula is a special case of [1], [16], [21]. This approach to the problem of counting lattice points in convex polytopes via the geometry of toric varieties is pursued in [7], [11], [12], [4] and [8].

However, we feel it is worthwhile, in the spirit of Khovanskii and Pukhlikov [17], to present an elementary proof in the context of polytopes. The use of generating functions simplifies further our approach in [3] where the case of simple polytopes with integral vertices was treated, based on the Euler relation for the number of faces of polytopes. The Riemann-Roch formula for toric varieties boils down to counting homogeneous monomials for various gradings on the algebra of polynomial functions in $N$ variables. This is what we achieve here in a direct way, and simultaneously for all gradings, by decomposing the generating function of $\mathcal{P}_{\Delta}(y, \lambda)$ into simple fractions. This approach is inspired by Jeffrey and Kirwan's proof of the Guillemin-Sternberg conjecture [14], based on iterated residues.

To state our main results in a precise way, we consider the vector space $W:=\mathbb{R}^{N}$ with standard basis $\left(w_{1}, \ldots, w_{N}\right)$, and the standard cone $C$ in $W$ generated by $w_{1}, \ldots, w_{N}$. For any subset $A$ of $W$, we denote by $[A]$ its characteristic function, i.e., the function with value 1 on $A$ and 0 outside $A$.

Define a surjective linear map $p: W \rightarrow E$ by $p\left(w_{k}\right)=\alpha_{k}$ for $1 \leq k \leq N$; then the kernel of $p$ is $V$. A subset $\sigma$ of $\{1, \ldots, N\}$ is called a basis of $\Delta$ if the sequence $\left(\alpha_{j}\right)_{j \in \sigma}$ is a basis of $E$. The set of bases of $\Delta$ is denoted by $\mathcal{B}(\Delta)$. For $\sigma \in \mathcal{B}(\Delta)$, we have a decomposition $W=V \oplus\left(\bigoplus_{j \in \sigma} \mathbb{R} w_{j}\right)$ and we denote by $\rho_{\sigma}: W \rightarrow V$ the corresponding projection. Then $\rho_{\sigma}(C)$ is a closed convex cone, generated by the basis $\left(\rho_{\sigma}\left(w_{k}\right)\right)_{k \notin \sigma}$ of $V$. Moreover, we have for any $k \notin \sigma: \rho_{\sigma}\left(w_{k}\right)=w_{k}-$ $\sum_{j \in \sigma} c_{j k} w_{j}$ where the real numbers $c_{j k}$ are defined by $\alpha_{k}=\sum_{j \in \sigma} c_{j k} \alpha_{j}$.

Choose a point $x \in C$ such that $p(x)$ does not lie on any hyperplane generated by some of the $\alpha_{k}$; then $x$ is called regular (in the case where $\operatorname{dim}(E)=1$, any non-zero $x$ is regular). For any basis $\sigma$, we can write $p(x)=\sum_{j \in \sigma} x_{\sigma, j} \alpha_{j}$ with nonzero coordinates $x_{\sigma, j}$. Let $\varepsilon_{\sigma, j}$ be the sign of $x_{\sigma, j}$, and let $\left|\sigma_{-}(x)\right|$ be the number 
of $j \in \sigma$ such that $\varepsilon_{\sigma, j}=-1$. Finally, let $C_{x}^{\sigma}$ be the closed convex cone generated by the $\varepsilon_{\sigma, j} w_{j}(j \in \sigma)$.

Theorem (decomposition into simple cones). The function

$$
[C]-\sum_{\sigma \in \mathcal{B}(\Delta)}(-1)^{\left|\sigma_{-}(x)\right|}\left[C_{x}^{\sigma}+\rho_{\sigma}(C)\right]
$$

is a linear combination of characteristic functions of closed convex cones which contain lines.

This result is proved in 1.2. It implies an exact formula for $[C]$ as a signed sum of characteristic functions of locally closed cones, see 1.4. By integrating over $C$ the exponential of a complex linear form on $W$, our decomposition implies the following formula.

Corollary (decomposition into simple fractions). For a generic complex linear form $z$ on $E$, and for generic complex numbers $y_{1}, \ldots, y_{N}$, we have

$$
\prod_{k=1}^{N} \frac{1}{y_{k}+\left\langle z, \alpha_{k}\right\rangle}=\sum_{\sigma \in \mathcal{B}(\Delta)}\left(\prod_{k \in \sigma} \frac{1}{y_{k}+\left\langle z, \alpha_{k}\right\rangle}\right)\left(\prod_{k \notin \sigma} \frac{1}{y_{k}-\sum_{j \in \sigma} c_{j k} y_{j}}\right) .
$$

This can be considered as a residue formula for the rational function $z \mapsto$ $\prod_{k=1}^{N}\left(y_{k}+\left\langle z, \alpha_{k}\right\rangle\right)^{-1}$. A direct proof of this formula is given in section 1.1, by a simple induction on $N$.

Actually, the assumption that $\alpha_{1}, \ldots, \alpha_{N}$ are contained in a lattice in $E$ is not needed for either of the two results above; but it will play a role in the next one, a residue formula for the periodic function $\prod_{k=1}^{N}\left(1-e^{y_{k}+\left\langle z, \alpha_{k}\right\rangle}\right)^{-1}$. Indeed, this assumption implies that the numbers $c_{j k}$ are rational, so that we can choose a common denominator $q$. For $\sigma \in \mathcal{B}(\Delta)$, denote by $G(\sigma, q)$ the finite abelian group

$$
(\mathbb{Z} / q \mathbb{Z})^{\sigma}=\left\{g=\left(g_{j}\right)_{j \in \sigma} \mid g_{j} \in \mathbb{Z} / q \mathbb{Z}\right\}
$$

endowed with characters $g \rightarrow e^{2 i \pi q^{-1} g_{j}}(j \in \sigma)$ and $g \rightarrow e^{2 i \pi \sum_{j \in \sigma} c_{j k} g_{j}}(k \notin \sigma)$.

Theorem (decomposition into simple fractions, the periodic case). Let $x \in C$ be regular. For any basis $\sigma$ of $\Delta$, write $p(x)=\sum_{j \in \sigma} x_{\sigma, j} \alpha_{j}$ and set $\varepsilon_{\sigma, j}:=\operatorname{sgn}\left(x_{\sigma, j}\right)$. Then, for a generic complex linear form $z$ on $E$, and for generic complex numbers $y_{1}, \ldots, y_{N}$, the product

$$
\prod_{k=1}^{N} \frac{1}{1-e^{y_{k}+\left\langle z, \alpha_{k}\right\rangle}}
$$

is equal to

$$
\sum_{\sigma \in \mathcal{B}(\Delta)} \sum_{g \in G(\sigma, q)} \prod_{j \in \sigma} \frac{q^{-1} \varepsilon_{\sigma, j}}{1-e^{q^{-1} \varepsilon_{\sigma, j}\left(2 i \pi g_{j}+y_{j}+\left\langle z, \alpha_{j}\right\rangle\right)}} \prod_{k \notin \sigma} \frac{1}{1-e^{y_{k}-\sum_{j \in \sigma} c_{j k}\left(y_{j}+2 i \pi g_{j}\right)}} .
$$

This formula is deduced in 2.3 from the decomposition of $C$ into simple cones, by summing the series $\sum_{w \in C \cap \mathbb{Z}^{N}} e^{w}$ and similar series for the cones $C_{x}^{\sigma}+\rho_{\sigma}(C)$. It may also be obtained directly by induction on $N$.

To apply these results to partition functions, we need the following notation. We consider the subdivision of $C(\Delta)$ given by the intersections of the cones $C(\sigma)$ (generated by the $\alpha_{j}, j \in \sigma$ ), for $\sigma \in \mathcal{B}(\Delta)$. The interiors of the maximal cones of 
this subdivision are called chambers. For such a chamber $\gamma$, we denote by $\mathcal{B}(\Delta, \gamma)$ the set of bases $\sigma$ such that $\gamma$ is contained in $C(\sigma)$.

Finally, for any basis $\sigma$, let $v_{\sigma}: E \rightarrow W$ be the linear map such that $v_{\sigma}\left(\alpha_{j}\right)=w_{j}$ for all $j \in \sigma$, and let $\mu(\sigma)$ be the volume of the parallelepiped $\left\{\sum_{j \in \sigma} t_{j} \alpha_{j} \mid 0 \leq\right.$ $\left.t_{j} \leq 1\right\}$. Then $\mu(\sigma)$ is the index of the subgroup $\bigoplus_{j \in \sigma} \mathbb{Z} \alpha_{j}$ in $\Lambda$. Let $G(\sigma)$ be the quotient $\left(\bigoplus_{j \in \sigma} \mathbb{Z} \alpha_{j}\right)^{*} / \Lambda^{*}$ where the star denotes the dual lattice. Then $G(\sigma)$ is a finite abelian group of order $\mu(\sigma)$, endowed with characters $e^{2 i \pi \lambda}$ for all $\lambda \in \Lambda$.

Theorem (closed formulae). Let $\gamma$ be a chamber.

(i) For all $h \in \bar{\gamma}$, the function

$$
\left[P_{\Delta}(h)\right]-\sum_{\sigma \in \mathcal{B}(\Delta, \gamma)}\left[v_{\sigma}(h)+\rho_{\sigma}(C)\right]
$$

is a linear combination of characteristic functions of convex polyhedra in $V(h)$, which contain lines.

(ii) For all $h \in \bar{\gamma}$, and for a generic linear form $y$ on $W$, we have

$$
\mathcal{V}_{\Delta}(y, h)=\sum_{\sigma \in \mathcal{B}(\Delta, \gamma)} \frac{e^{-\left\langle y, v_{\sigma}(h)\right\rangle}}{\mu(\sigma) \prod_{k \notin \sigma}\left(y_{k}-\sum_{j \in \sigma} c_{j k} y_{j}\right)} .
$$

In particular, we have

$$
\operatorname{vol}\left(P_{\Delta}(h)\right)=\sum_{\sigma \in \mathcal{B}(\Delta, \gamma)} \frac{\left\langle y, v_{\sigma}(h)\right\rangle^{n}}{n ! \mu(\sigma) \prod_{k \notin \sigma}\left(-y_{k}+\sum_{j \in \sigma} c_{j k} y_{j}\right)}
$$

so that the volume of $P_{\Delta}(h)$ is a polynomial function of $h$ on $\bar{\gamma}$.

(iii) For all $\lambda \in \bar{\gamma} \cap \Lambda$, and for a generic linear form $y$ on $W$, we have

$$
\mathcal{P}_{\Delta}(y, \lambda)=\sum_{\sigma \in \mathcal{B}(\Delta, \gamma)} \frac{e^{-\left\langle y, v_{\sigma}(\lambda)\right\rangle}}{\mu(\sigma)} \sum_{g \in G(\sigma)} e^{2 i \pi \lambda}(g) \prod_{k \notin \sigma}\left(1-e^{-2 i \pi \alpha_{k}}(g) e^{-y_{k}+\sum_{j \in \sigma} c_{j k} y_{j}}\right) .
$$

These formulae are proved in 3.2, 3.3 and 3.4 in a more general setting. They imply that the function $h \in \bar{\gamma} \mapsto \mathcal{V}_{\Delta}(y, h)$ extends to an analytic function $\mathcal{V}_{\Delta, \gamma}(y, h)$. Moreover, we can compute $\mathcal{P}_{\Delta}(y, \lambda)$ by applying to $\mathcal{V}_{\Delta, \gamma}(y, h)$ a series of differential operators, defined as follows.

For a complex number $a$, define $\operatorname{Todd}(a, z)$ as the expansion of

$$
\frac{z}{1-a e^{-z}}
$$

into a power series in $z$. For $\varphi$ a smooth function on $E$, and for $u \in E$, denote by $\partial(u) \varphi$ the derivative of $\varphi$ in the direction $u$. Then $\operatorname{Todd}(a, \partial(u))$ is a differential operator of infinite order, with constant coefficients. Moreover, for any complex number $t$, we have a series of differential operators with constant coefficients $\operatorname{Todd}(a, \partial(u)+t)$.

For $g \in E^{*} / \Lambda^{*}$ and $y=\left(y_{1}, \ldots, y_{N}\right)$, we define the Todd operator (a series of differential operators with constant coefficients) by

$$
\operatorname{Todd}_{\Delta}(g, \partial+y):=\prod_{k=1}^{N} \operatorname{Todd}\left(e^{-2 i \pi \alpha_{k}}(g), \partial\left(\alpha_{k}\right)+y_{k}\right) .
$$

Now we can state the following result, proved in 3.5 . 
Theorem (Euler-MacLaurin formula for partition functions). Let $\gamma$ be a chamber.

(i) The series

$$
\operatorname{Todd}_{\Delta}(g, \partial+y) \mathcal{V}_{\Delta, \gamma}(y, h)
$$

converges for all $g \in E^{*} / \Lambda^{*}$, and for small $y \in W^{*}$. Moreover, the sum of this series vanishes whenever $g$ is not in the finite set

$$
G(\gamma):=\bigcup_{\sigma \in \mathcal{B}(\Delta, \gamma)} G(\sigma)
$$

(ii) For any $\lambda \in \bar{\gamma} \cap \Lambda$, we have for small $y \in W^{*}$ :

$$
\mathcal{P}_{\Delta}(y, \lambda)=\left.\sum_{g \in G(\gamma)} e^{2 i \pi \lambda}(g)\left(\operatorname{Todd}_{\Delta}(g, \partial+y) \mathcal{V}_{\Delta, \gamma}(y, h)\right)\right|_{h=\lambda} .
$$

Setting $y=0$, we see that

$$
\mathcal{P}_{\Delta}(\lambda)=\sum_{g \in G(\gamma)} e^{2 i \pi \lambda}(g) \lim _{h \rightarrow \lambda, h \in \gamma} \operatorname{Todd}_{\Delta}(g, \partial) \operatorname{vol}\left(P_{\Delta}(h)\right) .
$$

In other words, the vector partition function is obtained by applying a canonical differential operator with periodic coefficients to the volume function. This result is new even for the classical partition function, that is, for the number of lattice points in rational simplices (formulae for the number of lattice points in lattice simplices are given in [7] and [9]).

By studying in sections 3.6 and 3.7 the partial derivatives of the function $\mathcal{V}_{\Delta, \gamma}$, we also express the sum of values of any polynomial function at all lattice points of the polytope $P_{\Delta}(\lambda)$ in terms of integrals, over the faces of this polytope, of the function and of its partial derivatives. In particular, the vector partition function is a linear combination of volumes of faces, with periodic coefficients. This refines results of $[20]$ and $[8]$.

Consider now a rational convex polytope $P$ in $\mathbb{R}^{n}$, that is, the convex hull of finitely many points with rational coordinates. Assume that the interior $P^{0}$ is not empty. We can define $P$ by a minimal set of inequalities

$$
P=\left\{v \in \mathbb{R}^{n} \mid\left\langle u_{k}, v\right\rangle+\lambda_{k} \geq 0,1 \leq k \leq N\right\}
$$

where $\left(u_{k}, \lambda_{k}\right) \in \mathbb{Z}^{n} \times \mathbb{Z}$ are primitive. Let $\mathcal{F}$ be the set of vectors $u_{k}, 1 \leq k \leq N$. Then $\mathcal{F}$ depends only on the directions of the faces of codimension one of $P$.

For $x \in \mathbb{R}^{N}$, we denote

$$
P(x)=\left\{v \in \mathbb{R}^{n} \mid\left\langle u_{k}, v\right\rangle+x_{k} \geq 0,1 \leq k \leq N\right\}
$$

and by $C(\mathcal{F})$ the closed convex cone in $\mathbb{R}^{N}$ such that $x \in C(\mathcal{F})$ if and only if $P(x)$ is not empty. Then $\lambda \in C(\mathcal{F})$ as $P=P(\lambda)$. In 4.1, we describe open chambers $\gamma \subset C(\mathcal{F})$ such that the closures $\bar{\gamma}$ cover $C(\mathcal{F})$. The choice of $\gamma$ such that $\lambda \in \bar{\gamma}$ amounts to the choice of an approximation of $P$ by the family of simple polytopes (with prescribed directions of faces) $P(x), x \in \gamma$. In particular, if $P$ is simple, then $\lambda$ is in a unique chamber $\gamma$.

Let $\varphi$ be a polynomial function on $\mathbb{R}^{n}$. Then the function

$$
x \mapsto I_{P}(\varphi)(x):=\int_{P(x)} \varphi(v) d v
$$

is piecewise polynomial on $C(\mathcal{F})$. More precisely, for each chamber $\gamma$, the function $x \in \bar{\gamma} \mapsto I_{P}(\varphi)(x)$ extends to a polynomial function $I_{P, \gamma}(\varphi)$ on $\mathbb{R}^{N}$, see 4.2 . 
For $g \in \mathbb{R}^{N}$, we write $(\lambda, g)$ for $\sum_{k=1}^{N} \lambda_{k} g_{k}$. We set

$$
\operatorname{Todd}(g, \partial / \partial x):=\prod_{k=1}^{N} \frac{\partial / \partial x_{k}}{1-e^{-2 i \pi g_{k}} e^{-\partial / \partial x_{k}}}
$$

This defines a differential operator of infinite order with constant coefficients: the Todd operator associated to $g$. It acts for example in the space of polynomial functions of $x=\left(x_{1}, \ldots, x_{N}\right)$.

Let $G(\mathcal{F})$ be the subset of $\left[0,1{ }^{N}\right.$ consisting of elements $\left(g_{k}\right)$ which satisfy the following conditions:

1) The vectors $u_{k} \in \mathcal{F}$ such that $g_{k} \neq 0$ are linearly independent.

2) $\sum_{k=1}^{N} g_{k} u_{k}$ is in $\mathbb{Z}^{n}$.

Then $G(\mathcal{F})$ is a finite set of rational points in $\mathbb{R}^{N}$.

Theorem (Euler-MacLaurin formula for rational polytopes). Let $P=P(\lambda)$ be an $n$-dimensional rational convex polytope in $\mathbb{R}^{n}$. Let $\varphi: \mathbb{R}^{n} \rightarrow \mathbb{C}$ be a polynomial function. Let $\gamma$ be a chamber such that $\lambda \in \bar{\gamma}$. Then

$$
\sum_{v \in P \cap \mathbb{Z}^{n}} \varphi(v)=\left.\sum_{g \in C(\mathcal{F})} e^{2 i \pi(\lambda, g)} \operatorname{Todd}(g, \partial / \partial x) I_{P, \gamma}(\varphi)(x)\right|_{x=\lambda} .
$$

Actually, we could restrict the sum over $C(\mathcal{F})$ to a smaller subset which depends on $\gamma$, see 4.4. In particular, for a simple polytope $P$ with integral vertices, the result above specializes to the main result of [3] (proved there by another method). If moreover the tangent cone at each vertex of $P$ is generated by a basis of $\mathbb{Z}^{n}$, we recover the Euler-MacLaurin formula of Khovanskii and Pukhlikov, see [17].

We thank the referee for his careful reading of our paper and for his comments and suggestions. The present version owes much to his recommendations, especially in sections 3.6 and 3.7 .

\section{DeCOMPOSItions INTO SIMPle CONES}

1.1. A decomposition into simple fractions. In this section, $E$ is a finitedimensional vector space over an infinite field $K$. Let $W$ be another finitedimensional $K$-vector space endowed with a surjective linear map $p: W \rightarrow E$. Let $\left(w_{1}, \ldots, w_{N}\right)$ be a basis of $W$, and set $\alpha_{k}=p\left(w_{k}\right)$ for $1 \leq k \leq N$. Then $\Delta=\left(\alpha_{1}, \ldots, \alpha_{N}\right)$ is a finite sequence of elements of $E$, which generates this vector space.

We denote by $\mathcal{B}(\Delta)$ the set of bases of $\Delta$, that is, of subsets $\sigma$ of $\{1,2, \ldots, N\}$ such that $\left(\alpha_{k}\right)_{k \in \sigma}$ form a basis of $E$. We denote by $K \sigma \subset W$ the vector space spanned by the $w_{k}(k \in \sigma)$, and by $V$ the kernel of $p$. Then $V$ is the space of linear relations between elements of $\Delta$. Moreover, we have a decomposition $W=K \sigma \oplus V$. Let us denote by $\rho_{\sigma}$ the projection from $W$ to $V$ determined by this decomposition.

We denote by $Q(W)$ the field of fractions of the symmetric algebra of $W$. Then $Q(W)$ is the field of rational functions on $W^{*}$.

Proposition. In $Q(W)$, we have the equality:

$$
\prod_{k=1}^{N} \frac{1}{w_{k}}=\sum_{\sigma \in \mathcal{B}(\Delta)}\left(\prod_{k \in \sigma} \frac{1}{w_{k}}\right)\left(\prod_{k \notin \sigma} \frac{1}{\rho_{\sigma}\left(w_{k}\right)}\right) .
$$


Proof. The proof is by induction on the dimension of $V$. If $\operatorname{dim} V=0$, then there is nothing to be proved. If $\operatorname{dim} V \geq 1$, let $r=\sum_{j=1}^{N} r_{j} w_{j}$ be a non-zero element of $V$. Then, multiplying $r$ by $r^{-1} \prod_{k=1}^{N} w_{k}^{-1}$, we obtain

$$
\prod_{k=1}^{N} \frac{1}{w_{k}}=\sum_{j, r_{j} \neq 0} \frac{r_{j}}{r}\left(\prod_{k \neq j} \frac{1}{w_{k}}\right) \text {. }
$$

Moreover, if $r_{j} \neq 0$, then the map $p$ restricts to a surjection

$$
p_{j}: W^{\hat{j}}=\bigoplus_{k \neq j} K w_{k} \rightarrow E
$$

and hence the kernel of $p_{j}$ is a hyperplane in $V$. Therefore, we can apply the induction hypothesis to $W^{\hat{j}}$, and we obtain, for each fixed $j$,

$$
\prod_{k \neq j} \frac{1}{w_{k}}=\sum_{\sigma, j \notin \sigma}\left(\prod_{k \in \sigma} \frac{1}{w_{k}}\right)\left(\prod_{k \notin \sigma, k \neq j} \frac{1}{\rho_{\sigma}\left(w_{k}\right)}\right) .
$$

It follows that

$$
\prod_{k=1}^{N} \frac{1}{w_{k}}=\sum_{\sigma}\left(\prod_{k \in \sigma} \frac{1}{w_{k}}\right) f_{\sigma}
$$

where we set

$$
f_{\sigma}=\sum_{j \notin \sigma} \frac{r_{j}}{r}\left(\prod_{k \notin \sigma \cup\{j\}} \frac{1}{\rho_{\sigma}\left(w_{k}\right)}\right) .
$$

Now, because $r=\sum_{k=1}^{N} r_{j} w_{j}$, and $\rho_{\sigma}\left(w_{j}\right)=0$ for all $j \in \sigma$, we have

$$
r=\sum_{j \notin \sigma} r_{j} \rho_{\sigma}\left(w_{j}\right) .
$$

Multiplying this equation by $r^{-1} \prod_{k \notin \sigma} \rho_{\sigma}\left(w_{k}\right)^{-1}$, we obtain

$$
f_{\sigma}=\prod_{k \notin \sigma} \frac{1}{\rho_{\sigma}\left(w_{k}\right)} \text {. }
$$

Let us rephrase the proposition in more concrete terms, by interpreting elements of $Q(W)$ as rational functions on $W^{*}$. We embed $E^{*}$ into $W^{*}$ via $p^{*}$; then, for generic $y \in W^{*}$, a function $\varphi$ in $Q(W)$ gives rise to a rational function $z \mapsto$ $\varphi\left(y+p^{*} z\right)$ on $E^{*}$. If moreover $\varphi$ is in the subring $Q(V)$, then $\varphi$ is constant on each coset $y+p^{*} E^{*}$ in $W^{*}$. So (1.1) translates into an identity of rational functions on $E^{*}$ which depend on the parameter $y$. To formulate it, we introduce the following notation.

For $\sigma \in \mathcal{B}(\Delta)$, there exist uniquely defined coefficients $c_{j k} \in K(j \in \sigma, k \notin \sigma)$ such that $\alpha_{k}=\sum_{j \in \sigma} c_{j k} \alpha_{j}$. Then $\rho_{\sigma}\left(w_{k}\right)=w_{k}-\sum_{j \in \sigma} c_{j k} w_{j}$.

Restricting (1.1) to $y+p^{*} E^{*}$ for generic $y \in W^{*}$, we obtain the following

Corollary. We have

$$
\prod_{k=1}^{N} \frac{1}{y_{k}+\left\langle z, \alpha_{k}\right\rangle}=\sum_{\sigma \in \mathcal{B}(\Delta)}\left(\prod_{k \in \sigma} \frac{1}{y_{k}+\left\langle z, \alpha_{k}\right\rangle}\right)\left(\prod_{k \notin \sigma} \frac{1}{y_{k}-\sum_{j \in \sigma} c_{j k} y_{j}}\right)
$$

for all $z \in E^{*}$ and $y_{1}, \ldots, y_{N} \in K$ such that the formula makes sense. 
1.2. A decomposition into simple cones. Let $W$ be a vector space of finite dimension $N$ over a subfield $K$ of the field of real numbers. A polyhedral cone in $W$ is a closed convex cone $C \subset W$ (with vertex at 0 ) which is generated by finitely many vectors $x_{1}, \ldots, x_{r}$; we set $C=C\left(x_{1}, \ldots, x_{r}\right)$. We denote by $[C]=\left[x_{1}, \ldots, x_{r}\right]$ the characteristic function of $C$, i.e., the function on $W$ with value 1 on $C$ and 0 outside $C$. We denote by $\mathcal{C}(W)$ the additive group of integral-valued functions on $W$, generated by all characteristic functions of polyhedral cones.

For any closed convex cone $C$, we denote by $C^{0}$ the relative interior of $C$, i.e., the interior of $C$ in the affine space generated by $C$. Observe that $\mathcal{C}(W)$ contains the characteristic functions of relative interiors of polyhedral cones, and more generally, of locally closed polyhedral cones. The subgroup of $\mathcal{C}(W)$ generated by characteristic functions of polyhedral cones which contain lines is denoted by $\mathcal{L} C(W)$. If $W=W_{1} \oplus W_{2}$ and $C_{j}$ is a polyhedral cone in $W_{j}$ for $j=1,2$, then we set $\left[C_{1}\right] *\left[C_{2}\right]=\left[C_{1}+C_{2}\right]$. We refer to [17] and to [19] for more properties of $\mathcal{C}(W)$ (which will not be used here).

Recall that a cone generated by linearly independent vectors is called simplicial. Let $C$ be a cone generated by a basis $\left(w_{1}, \ldots, w_{N}\right)$; then $C$ is simplicial of dimension $N$. Let $p: W \rightarrow E$ be a surjective linear map. We obtain an analog of our decomposition into simple fractions (proposition 1.1) for the image of the function $[C]$ in the quotient $\mathcal{C}(W) / \mathcal{L} C(W)$. To state it, we introduce the following notation.

For $C=C\left(w_{1}, \ldots, w_{N}\right)$ as before, the edges of $C$ are the half-lines generated by $w_{1}, \ldots, w_{N}$. The corresponding lines are called the axes of $C$. If $C^{\prime}$ is a simplicial cone having the same axes as $C$, then there exist well-defined signs $\varepsilon_{1}, \ldots, \varepsilon_{N}$ such that $C^{\prime}=C\left(\varepsilon_{1} w_{1}, \ldots, \varepsilon_{N} w_{N}\right)$. In this case, we set $\varepsilon\left(C, C^{\prime}\right)=\prod_{k=1}^{N} \varepsilon_{k}$.

For $1 \leq k \leq N$, we set $\alpha_{k}=p\left(w_{k}\right)$. Any basis $\sigma$ of $\Delta$ defines a simplicial cone

$$
C^{\sigma}:=C \cap K \sigma=C\left(w_{j}\right)_{j \in \sigma}
$$

in $K \sigma$, together with a simplicial cone

$$
\rho_{\sigma}(C)=C\left(\rho_{\sigma}\left(w_{k}\right)\right)_{k \notin \sigma}
$$

in $V$. A point $x \in W$ is called regular if $p(x)$ is not contained in any hyperplane of $E$ generated by some of the $\alpha_{k}$. For $x$ regular, we denote by $C_{x}^{\sigma}$ the unique cone having the same axes as $C^{\sigma}$, and such that $p\left(C_{x}^{\sigma}\right)$ meets $p(x)$. If $p(x)=\sum_{j \in \sigma} x_{\sigma, j} \alpha_{j}$, then no $x_{\sigma, j}$ is zero because $x$ is regular, and moreover

$$
C_{x}^{\sigma}=C\left(\operatorname{sgn}\left(x_{\sigma, j}\right) w_{j}\right)_{j \in \sigma} .
$$

Theorem. For any cone $C$ generated by a basis $\left(w_{1}, \ldots, w_{N}\right)$ of $W$, and for any regular element $x$ in $C$, we have

$$
[C]=\sum_{\sigma \in \mathcal{B}(\Delta)} \varepsilon\left(C^{\sigma}, C_{x}^{\sigma}\right)\left[C_{x}^{\sigma}\right] *\left[\rho_{\sigma}(C)\right]
$$

modulo $\mathcal{L} C(W)$.

1.3. Proof of theorem 1.2. The proof follows the same lines as the proof of proposition 1.1; we argue by induction over the dimension of $V$. If this dimension is zero, then $\Delta$ consists of linearly independent elements. In this case, there is a unique basis $\sigma=(1, \ldots, N)$ and $C=C_{x}^{\sigma}$ so there is nothing to be proved. 
If $\operatorname{dim}(V)=1$, then there is a non-trivial relation

$$
r=\sum_{j=1}^{N} r_{j} w_{j}
$$

We may assume that: $r_{1}, \ldots, r_{l}>0, r_{l+1}=\cdots=r_{m}=0$, and $r_{m+1}, \ldots, r_{N}<0$. Replacing the generating vectors $w_{j}$ by positive multiples, we may assume that $C=C\left(w_{1}, \ldots, w_{N}\right)$ and that

$$
r=\sum_{j=1}^{l} w_{j}-\sum_{j=m+1}^{N} w_{j}
$$

The bases of $\Delta$ are

$$
\sigma=\sigma_{j}:=(1, \ldots, j-1, j+1, \ldots, N)
$$

where $j \leq l$ or $j \geq m+1$. Let $x=\sum_{k=1}^{N} x_{k} w_{k}$ with non-negative coefficients $x_{1}, \ldots, x_{N}$. If $j \leq l$, the decomposition of $p(x)$ in the basis $\sigma_{j}$ is

$$
p(x)=\sum_{k=1, k \neq j}^{l}\left(x_{k}-x_{j}\right) \alpha_{k}+\sum_{k=l+1}^{m} x_{k} \alpha_{k}+\sum_{k=m+1}^{N}\left(x_{k}+x_{j}\right) \alpha_{k} .
$$

If $j \geq m+1$, the decomposition of $p(x)$ in the basis $\sigma_{j}$ is

$$
p(x)=\sum_{k=1}^{l}\left(x_{k}+x_{j}\right) \alpha_{k}+\sum_{k=l+1}^{m} x_{k} \alpha_{k}+\sum_{k=m+1, k \neq j}^{N}\left(x_{k}-x_{j}\right) \alpha_{k} .
$$

So $x$ is regular if and only if the numbers $x_{k}$ are pairwise distinct for $1 \leq k \leq l$ and for $m+1 \leq k \leq N$ as well, and $x_{k}>0$ for $l+1 \leq k \leq m$. Therefore, we may assume that

$$
x_{1}<\cdots<x_{l}, x_{m+1}>\cdots>x_{N} .
$$

Moreover, for $l<k \leq m$, the $k$-th coordinate of $p(x)$ on any basis of $\Delta$ is positive.

We have $\rho_{\sigma}\left(w_{j}\right)=r$ if $1 \leq j \leq l$, and $\rho_{\sigma}\left(w_{j}\right)=-r$ if $m+1 \leq j \leq N$. So the identity to be proved is

$$
\begin{aligned}
{\left[w_{1}, \ldots, w_{N}\right]=} & \sum_{j=1}^{l}(-1)^{j-1}\left[-w_{1}, \ldots,-w_{j-1}, w_{j+1}, \ldots, w_{N}, r\right] \\
& +\sum_{j=m+1}^{N}(-1)^{N-j}\left[w_{1}, \ldots, w_{j-1},-w_{j+1}, \ldots,-w_{N},-r\right]
\end{aligned}
$$

$\bmod \mathcal{L} C(W)$. To check it, we may assume that $l=m$.

Set $u_{k}=w_{k}$ for $1 \leq k \leq l$, and $u_{k}=-w_{k}$ for $l+1 \leq k \leq N$, so that $r=\sum_{k=1}^{N} u_{k}$. Set moreover

$$
u_{N+1}:=-r=-\sum_{k=1}^{N} u_{k} .
$$

Then the space $W$ is subdivided into the simplicial cones

$$
C\left(u_{1}, \ldots, u_{j-1}, u_{j+1}, \ldots, u_{N+1}\right)
$$


for $1 \leq j \leq N+1$. More precisely, $W$ is the disjoint union of locally closed cones

$$
\begin{gathered}
C_{0}:=C\left(u_{1}, \ldots, u_{l}\right)+C\left(u_{l+1}, \ldots, u_{N}\right)^{0} \\
C_{j}:=C\left(u_{1}, \ldots, u_{j-1}\right)^{0}+C\left(u_{j+1}, \ldots, u_{l}\right)+C\left(u_{l+1}, \ldots, u_{N+1}\right)^{0}(1 \leq j \leq l)
\end{gathered}
$$

and

$$
C_{j}:=C\left(u_{1}, \ldots, u_{l}\right)+C\left(u_{l+1}, \ldots, u_{j-1}\right)^{0}+C\left(u_{j+1}, \ldots, u_{N+1}\right)(l+1 \leq j \leq N)
$$

(this can be checked by starting with $C_{l+1}$ and adding successively $C_{l+2}, \ldots, C_{N}$, $\left.C_{0}, C_{1}, \ldots, C_{l}\right)$. It follows that

$$
\sum_{i=0}^{N}\left[C_{i}\right] \in \mathcal{L} C(W)
$$

Observe furthermore that, for any simplicial cone $C$, we have

$$
\left[C^{0}\right]-(-1)^{\operatorname{dim}(C)}[-C] \in \mathcal{L} C(W) .
$$

Indeed, it is enough to check this when $\operatorname{dim}(W)=1$; then $W$ is the disjoint union of $C^{0}$ and $-C$. So we can rewrite (1.3.2) as

$$
\begin{array}{r}
(-1)^{N-l}\left[w_{1}, \ldots, w_{N}\right]+\sum_{j=1}^{l}(-1)^{j+N-l}\left[-w_{1}, \ldots,-w_{j-1}, w_{j+1}, \ldots, w_{N}, r\right] \\
+\sum_{j=l+1}^{N}(-1)^{j-l-1}\left[w_{1}, \ldots, w_{j-1},-w_{j+1}, \ldots,-w_{N},-r\right] \in \mathcal{L} C(W) .
\end{array}
$$

This implies (1.3.1).

Finally, we consider the case where $\operatorname{dim}(V)$ is at least 2. We use the notation $C_{x / V}^{\sigma}$ for $C_{x}^{\sigma}$, to emphasize the dependence on $V$. Let $r=\sum_{j=1}^{N} r_{j} w_{j}$ be a non-zero element of $V$. Denote by $p^{r}: W \rightarrow W / K r$ the quotient map. We apply the first step of the proof to the data $C, x$ and $p^{r}$. Recall that each basis is the complement in $\{1, \ldots, N\}$ of some index $j$ such that $r_{j} \neq 0$. Denote by $\pi_{j}: W \rightarrow W^{\hat{j}}=$ $\bigoplus_{k \neq j} K w_{k}$ the corresponding projection with kernel $K r$. We set $C^{\hat{j}}=C \cap W^{\hat{j}}$ (a simplicial cone in $W^{\hat{j}}$ ) and we denote by $C_{x / r}^{\hat{j}}$ the unique simplicial cone having the same axes as $C^{\hat{j}}$ and containing $\pi_{j}(x)$. Then we have

$$
[C]=\sum_{j, r_{j} \neq 0} \varepsilon\left(C^{\hat{j}}, C_{x / r}^{\hat{j}}\right)\left[C_{x / r}^{\hat{j}}\right] *\left[\operatorname{sgn}\left(r_{j}\right) r\right]
$$

modulo $\mathcal{L} C(W)$. Because $r_{j} \neq 0$, the map $p$ restricts to a surjection $p_{j}: W^{\hat{j}} \rightarrow E$. So we can apply the induction hypothesis to the data $C_{x / r}^{\hat{j}}, \pi_{j}(x)$ and $p_{j}$. Observing that $p(x)=p_{j} \pi_{j}(x)$, we obtain, for each fixed $j$,

$$
\left[C_{x / r}^{\hat{j}}\right]=\sum_{\sigma, j \notin \sigma} \varepsilon\left(\left(C_{x / r}^{\hat{j}}\right)^{\sigma}, C_{x / V}^{\sigma}\right)\left[C_{x / V}^{\sigma}\right] *\left[\rho_{\sigma}\left(C_{x / r}^{\hat{j}}\right)\right]
$$

modulo $\mathcal{L} C\left(W^{\hat{j}}\right)$. It follows that

$$
[C]=\sum_{\sigma}\left[C_{x / V}^{\sigma}\right] * f_{\sigma}
$$


modulo $\mathcal{L} C(W)$, where we set

$$
f_{\sigma}:=\sum_{j, j \notin \sigma, r_{j} \neq 0} \varepsilon\left(C^{\hat{j}}, C_{x / r}^{\hat{j}}\right) \varepsilon\left(\left(C_{x / r}^{\hat{j}}\right)^{\sigma}, C_{x / V}^{\sigma}\right)\left[\rho_{\sigma}\left(C_{x / r}^{\hat{j}}\right)\right] *\left[\operatorname{sgn}\left(r_{j}\right) r\right]
$$

(an element of $\mathcal{C}(V)$ ). To end up the proof, we check that

$$
f_{\sigma}=\varepsilon\left(C^{\sigma}, C_{x / V}^{\sigma}\right)\left[\rho_{\sigma}(C)\right]
$$

modulo $\mathcal{L} C(V)$. For this, we apply the induction hypothesis to the simplicial cone $\left[\rho_{\sigma}(C)\right]$ in $V$, to the point $\rho_{\sigma}(x)$ in this cone, and to the quotient map $V \rightarrow V / K r$. Observe that

$$
r=\sum_{j \notin \sigma} r_{j} \rho_{\sigma}\left(w_{j}\right)
$$

So we obtain

$$
\left[\rho_{\sigma}(C)\right]=\sum_{j \notin \sigma, r_{j} \neq 0} \varepsilon\left(\rho_{\sigma}\left(C^{\hat{j}}\right), \rho_{\sigma}\left(C_{x / r}^{\hat{j}}\right)\right)\left[\rho_{\sigma}\left(C_{x / r}^{\hat{j}}\right)\right] *\left[\operatorname{sgn}\left(r_{j}\right) r\right]
$$

modulo $\mathcal{L} C(V)$. It remains to see that for each fixed $j$ and $\sigma$, with $j \notin \sigma$,

$$
\varepsilon\left(C^{\hat{j}}, C_{x / r}^{\hat{j}}\right) \varepsilon\left(\left(C_{x / r}^{\hat{j}}\right)^{\sigma}, C_{x / V}^{\sigma}\right)=\varepsilon\left(\rho_{\sigma}\left(C^{\hat{j}}\right), \rho_{\sigma}\left(C_{x / r}^{\hat{j}}\right)\right) \varepsilon\left(C^{\sigma}, C_{x / V}^{\sigma}\right) .
$$

Using the relation $\varepsilon\left(C, C^{\prime}\right)=\varepsilon\left(C, C^{\prime \prime}\right) \varepsilon\left(C^{\prime \prime}, C^{\prime}\right)$ between simplicial cones having the same axes, we reduce the proof to checking that

$$
\varepsilon\left(C^{\hat{j}}, C_{x / r}^{\hat{j}}\right) \varepsilon\left(\left(C_{x / r}^{\hat{j}}\right)^{\sigma}, C^{\sigma}\right)=\varepsilon\left(\rho_{\sigma}\left(C^{\hat{j}}\right), \rho_{\sigma}\left(C_{x / r}^{\hat{j}}\right)\right) .
$$

Writing $x=\sum_{k, k \neq j} y_{k} w_{k}+u r$, we have $C_{x / r}^{\hat{j}}=C\left(\operatorname{sgn}\left(y_{k}\right) w_{k}\right)_{k \neq j}$. Therefore, we have

$$
\varepsilon\left(C^{\hat{j}}, C_{x / r}^{\hat{j}}\right)=\prod_{k, k \neq j} \operatorname{sgn}\left(y_{k}\right)
$$

and moreover

$$
\varepsilon\left(\left(C_{x / r}^{\hat{j}}\right)^{\sigma}, C^{\sigma}\right)=\prod_{k \in \sigma} \operatorname{sgn}\left(y_{k}\right) .
$$

Finally, because $\rho_{\sigma}(x)=\sum_{k \notin \sigma, k \neq j} y_{k} \rho_{\sigma}\left(w_{k}\right)+u r$, we have

$$
\varepsilon\left(\rho_{\sigma}\left(C^{\hat{j}}\right), \rho_{\sigma}\left(C_{x / r}^{\hat{j}}\right)\right)=\prod_{k \notin \sigma, k \neq j} \operatorname{sgn}\left(y_{k}\right) .
$$

1.4. A decomposition into locally closed cones. Notation being as in 1.2 , we obtain decompositions of $[C]$ and of $\left[C^{0}\right]$ into alternated sums of characteristic functions of locally closed cones; both decompositions hold in $\mathcal{C}(W)$.

Let $x$ be a regular point of $W$. For $\sigma \in \mathcal{B}(\Delta)$, write $p(x)=\sum_{j \in \sigma} x_{\sigma, j} \alpha_{j}$ and denote by $\sigma_{+}(x)\left(\right.$ resp. $\left.\sigma_{-}(x)\right)$ the set of $j \in \sigma$ such that $x_{\sigma, j}>0$ (resp. $\left.<0\right)$. Then

$$
C_{x}^{\sigma}=C\left(w_{j}, j \in \sigma_{+}(x)\right) * C\left(-w_{j}, j \in \sigma_{-}(x)\right)
$$

and $\varepsilon\left(C^{\sigma}, C_{x}^{\sigma}\right)=(-1)^{\left|\sigma_{-}(x)\right|}$. We define locally closed polyhedral cones $C_{x}^{\prime \sigma}$ and $C_{x}^{\prime \prime \sigma}$ by

$$
C_{x}^{\prime \sigma}:=C\left(w_{j}, j \in \sigma_{+}(x)\right) * C\left(w_{j}, j \in \sigma_{-}(x)\right)^{0},
$$




$$
C_{x}^{\prime \prime \sigma}:=C\left(w_{j}, j \in \sigma_{+}(x)\right)^{0} * C\left(w_{j}, j \in \sigma_{-}(x)\right) .
$$

Both cones $C_{x}^{\prime \sigma}$ and $C_{x}^{\prime \prime \sigma}$ are contained in $C$.

A linear form $y$ on $W$ is called regular if $y\left(w_{k}\right) \neq 0$ for $1 \leq k \leq N$, and $y\left(\rho_{\sigma}\left(w_{k}\right)\right) \neq 0$ for all $\sigma \in \mathcal{B}(\Delta)$ and all $k \notin \sigma$. Such a form exists as all vectors $\rho_{\sigma}\left(w_{k}\right), k \notin \sigma$ are non-zero. For regular $y$, we denote by $\sigma_{+}^{c}(y)$ (resp. $\left.\sigma_{-}^{c}(y)\right)$ the set of $k$ such that $1 \leq k \leq N, k \notin \sigma$ and $y\left(\rho_{\sigma}\left(w_{k}\right)\right)>0$ (resp. $\left.<0\right)$. We set

$$
\begin{aligned}
& \rho_{\sigma}(C)_{y}^{\prime}:=C\left(\rho_{\sigma}\left(w_{k}\right), k \in \sigma_{+}^{c}(y)\right) * C\left(-\rho_{\sigma}\left(w_{k}\right), k \in \sigma_{-}^{c}(y)\right)^{0}, \\
& \rho_{\sigma}(C)_{y}^{\prime \prime}:=C\left(\rho_{\sigma}\left(w_{k}\right), k \in \sigma_{+}^{c}(y)\right)^{0} * C\left(-\rho_{\sigma}\left(w_{k}\right), k \in \sigma_{-}^{c}(y)\right) .
\end{aligned}
$$

Both cones $\rho_{\sigma}(C)_{y}^{\prime}$ and $\rho_{\sigma}(C)_{y}^{\prime \prime}$ are contained in the half-space $y \geq 0$.

Theorem. Let $C$ be a cone generated by a basis $\left(w_{1}, \ldots, w_{N}\right)$ of $W$. Then, for regular $x \in C$ and regular $y \in V^{*}$ such that $y \geq 0$ on $C$, we have

$$
\begin{aligned}
{[C] } & =\sum_{\sigma \in \mathcal{B}(\Delta)}(-1)^{\left|\sigma_{-}^{c}(y)\right|}\left[C_{x}^{\prime \sigma}\right] *\left[\rho_{\sigma}(C)_{y}^{\prime}\right], \\
{\left[C^{0}\right] } & =\sum_{\sigma \in \mathcal{B}(\Delta)}(-1)^{\left|\sigma_{-}^{c}(y)\right|}\left[C_{x}^{\prime \prime \sigma}\right] *\left[\rho_{\sigma}(C)_{y}^{\prime \prime}\right] .
\end{aligned}
$$

Proof. Observe that

$$
\left[C_{x}^{\sigma}\right]=(-1)^{\left|\sigma_{-}(x)\right|}\left[C_{x}^{\prime \sigma}\right]
$$

modulo $\mathcal{L} C(W)$, and that

$$
\left[\rho_{\sigma}(C)\right]=(-1)^{\left|\sigma_{-}^{c}(y)\right|}\left[\rho_{\sigma}(C)_{y}^{\prime}\right]
$$

modulo $\mathcal{L} C(V)$. Combined with theorem 1.2, this implies that the function

$$
[C]-\sum_{\sigma \in \mathcal{B}(\Delta)}(-1)^{\left|\sigma_{-}^{c}(y)\right|}\left[C_{x}^{\prime \sigma}\right] *\left[\rho_{\sigma}(C)_{y}^{\prime}\right]=: f
$$

is in $\mathcal{L} C(W)$. On the other hand, $f$ is an alternated sum of characteristic functions of (closed) polyhedral cones $C_{j}$ such that each $C_{j} \backslash\{0\}$ is contained in the open half-space $y>0$. Therefore, $f$ is supported in some acute cone (i.e. in some closed convex cone which contains no line).

To conclude the proof of (1.4.1), we check that a function $f \in \mathcal{L} C(W)$ identically 0 outside an acute cone $D$ must be zero. Let $\mathcal{F}(W)$ be the vector space of functions on $W$. We embed $\mathcal{C}(W)$ into $\mathcal{F}(W)$. The additive group of $W$ acts on $\mathcal{F}(W)$ by translations; we denote by $w \mapsto T(w)$ this action. Observe that, for a polyhedral cone $C$ which contains a line $\ell$, we have $(1-T(x))[C]=0$ for all $x \in \ell$. Because $f \in \mathcal{L} C(W)$, it follows that there exist $x_{1}, \ldots, x_{r} \in W \backslash\{0\}$ such that

$$
\prod_{j=1}^{r}\left(1-T\left(t_{j} x_{j}\right)\right) f=0
$$

for all $t_{j} \in K$. Moreover, we can find $z \in W^{*}$ such that $z>0$ on $D \backslash\{0\}$ and that $\left\langle z, x_{j}\right\rangle \neq 0$ for all $j$. Replacing $x_{j}$ by $-x_{j}$, we may assume that $\left\langle z, x_{j}\right\rangle<0$ for all $j$. Let $w \in W$. We can choose $A>0$ such that

$$
\left\langle z, w+\sum_{j \in J} t_{j} x_{j}\right\rangle<0
$$


for any non-empty subset $J$ of $\{1, \ldots, r\}$ and for $t_{j}>A$. Therefore, we have

$$
0=\left(\prod_{j=1}^{r}\left(1-T\left(-t_{j} x_{j}\right)\right) f\right)(w)=\sum_{J \subset\{1, \ldots, r\}}(-1)^{|J|} f\left(w+\sum_{j \in J} t_{j} x_{j}\right) .
$$

By assumption, our function $f$ is identically 0 on the open half-space $z<0$. It follows that $f(w)=0$.

To derive (1.4.2), we apply (1.4.1) to the cone $-C=C\left(-w_{1}, \ldots,-w_{N}\right)$ with regular points $-x \in-C$ and $-y \in-C^{\vee}$. Then we obtain

$$
[-C]=\sum_{\sigma \in \mathcal{B}(\Delta)}(-1)^{\left|\sigma_{-}^{c}(y)\right|}\left[-C_{x}^{\prime \sigma}\right] *\left[-\rho_{\sigma}(C)_{y}^{\prime}\right]
$$

Using (1.3.3), we obtain that the function

$$
g:=\left[C^{0}\right]-\sum_{\sigma \in \mathcal{B}(\Delta)}(-1)^{\left|\sigma_{-}^{c}(y)\right|}\left[C_{x}^{\prime \prime \sigma}\right] *\left[\rho_{\sigma}(C)_{y}^{\prime \prime}\right]
$$

is in $\mathcal{L} C(W)$. But $g$ vanishes identically outside some acute cone, and hence $g=0$.

Observing that $\left[\rho_{\sigma}(C)_{y}^{\prime}\right]-(-1)^{\left|\sigma_{-}^{c}(y)\right|}\left[\rho_{\sigma}(C)\right]$ and $\left[\rho_{\sigma}(C)_{y}^{\prime \prime}\right]-(-1)^{\left|\sigma_{-}^{c}(y)\right|}\left[\rho_{\sigma}(C)^{0}\right]$ are in $\mathcal{L} C(V)$, we obtain the following

Corollary. Let $C$ be a cone generated by a basis of $W$. Then, for regular $x \in C$, we have modulo the subgroup of $\mathcal{C}(W)$ generated by polyhedral cones which contain lines of $V$ :

$$
\begin{aligned}
{[C] } & =\sum_{\sigma \in \mathcal{B}(\Delta)}\left[C_{x}^{\prime \sigma}\right] *\left[\rho_{\sigma}(C)\right], \\
{\left[C^{0}\right] } & =\sum_{\sigma \in \mathcal{B}(\Delta)}\left[C_{x}^{\prime \prime \sigma}\right] *\left[\rho_{\sigma}(C)^{0}\right] .
\end{aligned}
$$

In fact, (1.4.3) and (1.4.4) are exchanged by the involution which maps $[C]$ to the function $(-1)^{\operatorname{dim}(C)}\left[C^{0}\right]$ for any polyhedral cone $C$ in $W$, see [19], 3.3.

1.5. Laplace transforms and polyhedral cones. In this section, we establish a connection between our results in 1.1 and 1.2, via the Laplace transforms of polyhedral cones.

We consider a real vector space $W$ of dimension $N$, endowed with a Lebesgue measure $d w$. Let $C \subset W$ be an acute polyhedral cone, and let $C^{\vee} \subset W^{*}$ be its dual cone. Then the interior of $C^{\vee}$ is not empty. Moreover, for each $z \in W_{\mathbb{C}}$ such that $\operatorname{Re}(z)$ is in the interior of $C^{\vee}$, the integral

$$
R(C)(z):=\int_{C} e^{-\langle w, z\rangle} d w
$$

converges, and defines the Laplace transform of $C$.

First properties of the Laplace transform are given by the proposition below, whose easy proof is omitted. A closed formula for the Laplace transform of any acute polyhedral cone is obtained in 4.2 . 
Proposition. (i) Let $C$ be the cone generated by a basis $\left(w_{1}, \ldots, w_{N}\right)$ of $W$. Then

$$
R(C)(z)=\operatorname{vol}|w| \prod_{j=1}^{N} \frac{1}{\left\langle z, w_{j}\right\rangle}
$$

where vol $|w|$ denotes the volume of the parallelepiped $\left\{\sum_{j=1}^{N} t_{j} w_{j} \mid 0 \leq t_{j} \leq 1\right\}$.

(ii) For any acute polyhedral cone $C$, the map $z \rightarrow R(C)(z)$ extends to a rational function $R(C)$ on $W_{\mathbb{C}}^{*}$.

(iii) The map $C \rightarrow R(C)$ extends to an additive map $R: \mathcal{C}(W) \rightarrow Q\left(W_{\mathbb{C}}\right)$ which vanishes on $\mathcal{L} C(W)$ and also on functions with support in a set of measure 0 .

Consider now a cone $C$ generated by a basis $\left(w_{1}, \ldots, w_{N}\right)$, and a regular point $x$ in $C$. Applying $R$ to the identity (1.2) and using (1.5), we recover identity (1.1). In other words, theorem 1.2 implies proposition 1.1. But theorem 1.2 is stronger than proposition 1.1, which would follow from an identity modulo the subgroup of $\mathcal{C}(W)$ generated by $\mathcal{L} C(W)$ and by characteristic functions of cones of smaller dimension.

\section{Residue FORMUlae FOR PERIODiC FUnCtions}

2.1. Formal series and rational functions associated to polyhedral cones. We maintain the notation of 1.2 , and we assume that $K$ is the field of rational numbers. Let $L$ be a lattice in $W$. The group algebra of $L$ over $\mathbb{Z}$ is denoted by $\mathbb{Z}[L]$, with canonical basis $\left(e^{l}\right)_{l \in L}$ and with quotient field $\mathbb{Q}(L)$. The choice of a basis $\left(w_{1}, \ldots, w_{N}\right)$ of $L$ identifies $\mathbb{Z}[L]$ to the ring of Laurent polynomials in the indeterminates $e^{w_{k}}(1 \leq k \leq N)$, with integral coefficients, and $\mathbb{Q}(L)$ to the field of rational functions in these indeterminates, with rational coefficients.

We denote by $\mathbb{Z}[[L]]$ the space of formal series $\sum_{l \in L} a_{l} e^{l}$ where $\left(a_{l}\right)_{l \in L}$ is a family of integers. Then $\mathbb{Z}[[L]]$ is a module over $\mathbb{Z}[L]$, multiplication by $e^{l}$ being defined by $e^{l} \sum_{m \in L} e^{m}=\sum_{m \in L} a_{m-l} e^{m}$. A formal series $f$ is called summable if there exist non-zero vectors $u_{1}, \ldots, u_{r} \in L$ such that $\left(\prod_{k=1}^{r}\left(1-e^{u_{k}}\right)\right) f=: P$ is in $\mathbb{Z}[L]$. Then the element $P \prod_{k=1}^{r}\left(1-e^{u_{k}}\right)^{-1}$ of $\mathbb{Q}(L)$ is independent of the choices of $u_{1}, \ldots, u_{r}$, and is called the sum of $f$.

As an example, for any non-zero $w \in L$, the series $\sum_{k=0}^{\infty} e^{k w}$ is summable with sum $1 /\left(1-e^{w}\right)$, while the series $\sum_{k=-\infty}^{\infty} e^{k w}$ is summable with sum zero.

We recall the following well-known result (see [13], Theorem 1.2, [4]).

Proposition. (i) For any polyhedral cone $C$ in $W$, the formal series

$$
f(C, L):=\sum_{l \in C \cap L} e^{l}
$$

is summable; let $F(C, L)$ be its sum.

(ii) The cone $C$ is acute if and only if $F(C, L) \neq 0$.

In fact, for acute $C$, the rational function $F(C, L)$ can be interpreted as follows: For any $y$ in the interior of the dual cone of $C$, the series

$$
\sum_{w \in C \cap L} e^{-\langle y, w\rangle}
$$

is convergent, and its sum is $F(C, L)(-y)$. In other words, the "algebraic" sum of $f(C, L)$ coincides with the summation of the series wherever it makes sense. 
For example, if $C$ is the cone generated by a basis $\left(w_{1}, \ldots, w_{N}\right)$ of the lattice $L$, then

$$
f(C, L)=\sum_{k_{1}, \ldots, k_{N} \geq 0} e^{k_{1} w_{1}+\cdots+k_{N} w_{N}}
$$

and hence

$$
F(C, L)=\prod_{j=1}^{N} \frac{1}{1-e^{w_{j}}}
$$

Observe that the map $C \mapsto f(C, L)$ defines an additive map

$$
\mathcal{C}(W) \rightarrow \mathbb{Z}[[L]]: \varphi \mapsto \sum_{l \in L} \varphi(l) e^{l} .
$$

It follows that the map $C \mapsto F(C, L)$ defines an additive map $\mathcal{C}(W) \rightarrow \mathbb{Q}(L)$ which vanishes on $\mathcal{L} C(W)$.

Consider now a lattice $M$ in $W$ which contains $L$, and a polyhedral cone $C$ in $W$. We express $F(C, L)$ in terms of $F(C, M)$. For this, denote by $L^{*}, M^{*}$ the lattices in $W^{*}$ dual to $L, M$. Then $M^{*}$ is contained in $L^{*}$ and the quotient $L^{*} / M^{*}$ is a finite subgroup of $W^{*} / M^{*}$ of order $[M: L]$. Each $m \in M$ defines a character

$$
e^{2 i \pi m}: W^{*} / M^{*} \rightarrow \mathbb{C}^{*} .
$$

We let $L^{*} / M^{*}$ act on $\mathbb{C}[M], \mathbb{C}[[M]]$ and $\mathbb{C}(M)$ by

$$
g \cdot e^{m}=e^{2 i \pi m}(g) e^{m} .
$$

Then we have

$$
\frac{1}{[M: L]} \sum_{g \in L^{*} / M^{*}} g \cdot e^{m}= \begin{cases}e^{m} & \text { if } m \in L \\ 0 & \text { otherwise }\end{cases}
$$

It follows that

$$
f(C, L)=\frac{1}{[M: L]} \sum_{g \in L^{*} / M^{*}} g \cdot f(C, M)
$$

Now let $D=\prod_{k=1}^{r}\left(1-e^{u_{k}}\right)$ be a non-zero element in $\mathbb{Z}[M]$ such that $\operatorname{Df}(C, M)$ is in $\mathbb{Z}[M]$. Observe that $D(q):=\prod_{k=1}^{r}\left(1-e^{q u_{k}}\right)$ has the same property, for all non-zero integers $q$. Choose $q$ such that $q M \subset L$; then, multiplying by $D(q) \in \mathbb{Z}[L]$ we obtain:

$$
F(C, L)=\frac{1}{[M: L]} \sum_{g \in L^{*} / M^{*}} g \cdot F(C, M) .
$$

In particular, for a cone $C$ generated by a basis $\left(m_{1}, \ldots, m_{N}\right)$ of $M$, we have

$$
F(C, L)=\frac{1}{[M: L]} \sum_{g \in L^{*} / M^{*}} \prod_{k=1}^{N} \frac{1}{1-e^{2 i \pi m_{k}}(g) e^{m_{k}}}
$$


2.2. A decomposition into simple fractions. We maintain the notation of 1.2 and 2.1; we give a decomposition into simple fractions of the function $F(C, L)$ for a cone $C$ generated by a basis $w_{1}, \ldots, w_{N}$ of $L$. Then

$$
F(C, L)=\prod_{k=1}^{N} \frac{1}{1-e^{w_{k}}} .
$$

Let $x$ be regular in $C$. For each basis $\sigma$ of $\Delta$, write $p(x)=\sum_{j \in \sigma} x_{\sigma, j} \alpha_{j}$ and set $\varepsilon_{\sigma, j}=\operatorname{sgn}\left(x_{\sigma, j}\right)$. By theorem 1.2, we have

$$
F(C, L)=\sum_{\sigma \in \mathcal{B}(\Delta)}\left(\prod_{j \in \sigma} \varepsilon_{\sigma, j}\right) F\left(C_{x}^{\sigma}+\rho_{\sigma}(C), L\right) .
$$

We now compute each $F\left(C_{x}^{\sigma}+\rho_{\sigma}(C), L\right)$. We set $\Lambda:=p(L)$; then $\Lambda$ is a lattice in $E$. In the case where $\left(\alpha_{j}\right)_{j \in \sigma}$ is a basis of $\Lambda$, it follows that the $w_{j}(j \in \sigma)$ and the $\rho_{\sigma}\left(w_{k}\right)(k \notin \sigma)$ are a basis of $L$. Then we have

$$
F\left(C_{x}^{\sigma}+\rho_{\sigma}(C), L\right)=\prod_{j \in \sigma} \frac{1}{1-e^{\varepsilon_{\sigma, j} w_{j}}} \prod_{k \notin \sigma} \frac{1}{1-e^{\rho_{\sigma}\left(w_{k}\right)}} .
$$

In the general case, the formula is slightly more complicated and involves sums with roots of unity, defined as follows.

Denote by $\Lambda^{*} \subset E^{*}$ the dual lattice of $\Lambda$. Then each $\lambda \in \Lambda$ defines a character

$$
e^{2 i \pi \lambda}: E^{*} / \Lambda^{*} \rightarrow \mathbb{C}^{*}
$$

For any basis $\sigma$, we set

$$
G(\sigma):=\left\{g \in E^{*} / \Lambda^{*} \mid e^{2 i \pi \alpha_{j}}(g)=1 \forall j \in \sigma\right\} .
$$

Then $G(\sigma)=\left(\bigoplus_{j \in \sigma} \mathbb{Z} \alpha_{j}\right)^{*} / \Lambda^{*}$ is a finite subgroup of $E^{*} / \Lambda^{*}$. We denote by $\mu(\sigma)$ the order of $G(\sigma)$, so that

$$
\mu(\sigma)=\left[\Lambda: \bigoplus_{j \in \sigma} \mathbb{Z} \alpha_{j}\right] .
$$

In particular, $\mu(\sigma)=1$ if and only if $\left(\alpha_{j}\right)_{j \in \sigma}$ is a basis of $\Lambda$.

We set

$$
L_{\sigma}:=p^{-1}(\Lambda) \cap \mathbb{Q} \sigma .
$$

This defines a lattice in $\mathbb{Q} \sigma$. We let $G(\sigma)$ act on $\mathbb{C}\left[L_{\sigma}\right], \mathbb{C}\left[\left[L_{\sigma}\right]\right]$ and $\mathbb{C}\left(L_{\sigma}\right)$ by

$$
g \cdot e^{m}:=e^{2 i \pi p(m)}(g) e^{m}
$$

(this makes sense because $p\left(L_{\sigma}\right) \subset \Lambda$ ).

Theorem. Let $C$ be a cone generated by a basis $\left(w_{1}, \ldots, w_{N}\right)$ of L. Let $x \in C$ be regular; for each basis $\sigma$, write $p(x)=\sum_{j \in \sigma} x_{\sigma, j} \alpha_{j}$ and set $\varepsilon_{\sigma, j}=\operatorname{sgn}\left(x_{\sigma, j}\right)$. Then we have

$$
\prod_{k=1}^{N} \frac{1}{1-e^{w_{k}}}=\sum_{\sigma \in \mathcal{B}(\Delta)} \frac{\left(\prod_{j \in \sigma} \varepsilon_{\sigma, j}\right)}{\mu(\sigma)} \sum_{g \in G(\sigma)} \frac{g \cdot F\left(C_{x}^{\sigma}, L_{\sigma}\right)}{\prod_{k \notin \sigma}\left(1-e^{-2 i \pi \alpha_{k}}(g) e^{\rho_{\sigma}\left(w_{k}\right)}\right)} .
$$

Proof. We need to check that each $F\left(C_{x}^{\sigma}+\rho_{\sigma}(C), L\right)$ is equal to

$$
\frac{1}{\mu(\sigma)} \sum_{g \in G(\sigma)} \frac{g \cdot F\left(C_{x}^{\sigma}, L_{\sigma}\right)}{\prod_{k \notin \sigma}\left(1-e^{-2 i \pi \alpha_{k}}(g) e^{\rho_{\sigma}\left(w_{k}\right)}\right)} .
$$


We set:

$$
M_{\sigma}:=L_{\sigma} \oplus \rho_{\sigma}(L) .
$$

Then $M_{\sigma}$ is a lattice in $W$. Moreover, we have

$$
M_{\sigma}=L_{\sigma}+L .
$$

Indeed, for any $w \in L$, we have $w=\left(w-\rho_{\sigma}(w)\right)+\rho_{\sigma}(w)$ with $w-\rho_{\sigma}(w) \in$ $p^{-1}(\Lambda) \cap \mathbb{Q} \sigma$ and $\rho_{\sigma}(w) \in \rho_{\sigma}(L)$. Clearly, we have

$$
F\left(C_{x}^{\sigma}+\rho_{\sigma}(C), M_{\sigma}\right)=\frac{F\left(C_{x}^{\sigma}, L_{\sigma}\right)}{\prod_{k \notin \sigma}\left(1-e^{\rho_{\sigma}\left(w_{k}\right)}\right)} .
$$

To finish the proof by applying (2.1.1), we have to describe the quotient group $L^{*} / M_{\sigma}^{*}$. Consider the map $p_{\sigma}: W \rightarrow E$ defined by $p_{\sigma}\left(w_{j}\right)=\alpha_{j}$ if $j \in \sigma$, and $p_{\sigma}\left(w_{k}\right)=0$ for $k \notin \sigma$. Then $p_{\sigma}\left(\rho_{\sigma}\left(w_{k}\right)\right)=-\alpha_{k}$ for all $k \notin \sigma$, and moreover $p_{\sigma}$ coincides with $p$ on $L_{\sigma}$. It follows that $p_{\sigma}\left(M_{\sigma}\right)=\Lambda$. Because $p_{\sigma}(L)=\bigoplus_{j \in \sigma} \mathbb{Z} \alpha_{j}$, the induced map $M_{\sigma} / L \rightarrow \Lambda / \bigoplus_{j \in \sigma} \mathbb{Z} \alpha_{j}$ is an isomorphism. By this isomorphism, the group $L^{*} / M_{\sigma}^{*}$ is identified to $G(\sigma)$, and we conclude by (2.1.2).

2.3. One more decomposition into simple fractions, and a residue formula for periodic functions. We give another decomposition of the function $\prod_{k=1}^{N} \frac{1}{1-e^{w_{k}}}$ into simple fractions, which involves sums over a unique group of roots of unity. Then we specialize our identity in the variables $e^{w_{k}}$ into a residue formula for periodic functions.

Choose a positive integer $q$ such that $q \Lambda$ is contained in $\bigoplus_{j \in \sigma} \mathbb{Z} \alpha_{j}=: \mathbb{Z} \sigma$ for all bases $\sigma$, that is, $q c_{j k}$ is an integer for all $j \in \sigma$ and all $k \notin \sigma$ (recall that the $c_{j k}$ are defined by $\left.\alpha_{k}=\sum_{j \in \sigma} c_{j k} \alpha_{j}\right)$. For $\sigma \in \mathcal{B}(\Delta)$, set

$$
G(\sigma, q):=(\mathbb{Z} / q \mathbb{Z})^{\sigma}=\left\{g=\left(g_{j}\right)_{j \in \sigma} \mid g_{j} \in \mathbb{Z} / q \mathbb{Z}\right\} .
$$

Then each $j \in \sigma$ defines a character

$$
g \mapsto e^{2 i \pi q^{-1} g_{j}}
$$

of the finite group $G(\sigma, q)$. Moreover, for all $k \notin \sigma$, we have a well-defined character

$$
g \mapsto e^{2 i \pi \sum_{j \in \sigma} c_{j k} g_{j}}
$$

because the $q c_{j k}$ are integers.

Observe that $G(\sigma, q) \simeq(\mathbb{Z} \sigma)^{*} /\left(\mathbb{Z} q^{-1} \sigma\right)^{*}$ surjects onto $G(\sigma)=(\mathbb{Z} \sigma)^{*} / \Lambda^{*}$. Moreover, for $k \notin \sigma$, the character $e^{2 i \pi \alpha_{k}}$ of $G(\sigma)$ gives rise to the character $e^{2 i \pi \sum_{j \in \sigma} c_{j k} g_{j}}$ of $G(\sigma, q)$.

Theorem. Let $C$ be a cone generated by a basis $\left(w_{1}, \ldots, w_{N}\right)$ of $L$. Let $x \in C$ be regular; for each basis $\sigma$, write $p(x)=\sum_{j \in \sigma} x_{\sigma, j} \alpha_{j}$ and set $\varepsilon_{\sigma, j}=\operatorname{sgn}\left(x_{\sigma, j}\right)$. Then the product

$$
\prod_{k=1}^{N} \frac{1}{1-e^{w_{k}}}
$$

is equal to

$$
\sum_{\sigma \in \mathcal{B}(\Delta)} \sum_{g \in G(\sigma, q)} \prod_{j \in \sigma} \frac{q^{-1} \varepsilon_{\sigma, j}}{1-e^{q^{-1} \varepsilon_{\sigma, j}\left(w_{j}+2 i \pi g_{j}\right)}} \prod_{k \notin \sigma} \frac{1}{1-e^{w_{k}-\sum_{j \in \sigma} c_{j k}\left(w_{j}+2 i \pi g_{j}\right)}} .
$$


Proof. For each $\sigma$, consider

$$
M_{\sigma, q}=\bigoplus_{j \in \sigma} \mathbb{Z} q^{-1} w_{j} \oplus \rho_{\sigma}(L) .
$$

Then

$$
M_{\sigma, q}=\bigoplus_{j \in \sigma} \mathbb{Z} q^{-1} w_{j}+L
$$

Clearly, we have

$$
F\left(C_{x}^{\sigma}+\rho_{\sigma}(C), M_{\sigma, q}\right)=\frac{1}{\prod_{j \in \sigma}\left(1-e^{q^{-1} \epsilon_{\sigma, j} w_{j}}\right)} \frac{1}{\prod_{k \notin \sigma}\left(1-e^{\rho_{\sigma}\left(w_{k}\right)}\right)} .
$$

Moreover, the map $p_{\sigma}$ sends $M_{\sigma, q}$ onto $q^{-1} \mathbb{Z} \sigma$ and hence $p_{\sigma}^{*}$ induces an isomorphism $G(\sigma, q) \rightarrow L^{*} / M_{\sigma, q}^{*}$. This isomorphism identifies the character $g \mapsto e^{2 i \pi q^{-1} g_{j}}$ to $e^{2 i \pi q^{-1} w_{j}}$, and $g \mapsto e^{-2 i \pi \sum_{j \in \sigma} c_{j k} g_{j}}$ to $e^{2 i \pi\left(\rho_{\sigma}\left(w_{k}\right)-w_{k}\right)}=e^{2 i \pi \rho_{\sigma}\left(w_{k}\right)}$. We conclude as in 2.2 .

Example. Let us use the notation of 1.3. If the space of relations between the elements $\alpha_{k}=p\left(w_{k}\right)$ is generated by

$$
r=\sum_{j=1}^{l} w_{j}-\sum_{j=m+1}^{N} w_{j}
$$

then the identity to be proven is

$$
\begin{gathered}
\prod_{k=1}^{N} \frac{1}{1-e^{w_{k}}}=\sum_{j=1}^{l}(-1)^{j-1} \frac{1}{1-e^{-w_{1}}} \cdots \frac{1}{1-e^{-w_{j-1}}} \frac{1}{1-e^{w_{j+1}}} \cdots \frac{1}{1-e^{w_{N}}} \frac{1}{1-e^{r}} \\
\quad+\sum_{j=m+1}^{N}(-1)^{N-j} \frac{1}{1-e^{w_{1}}} \cdots \frac{1}{1-e^{w_{j-1}}} \frac{1}{1-e^{-w_{j+1}}} \cdots \frac{1}{1-e^{-w_{N}}} \frac{1}{1-e^{-r}}
\end{gathered}
$$

which is easily checked directly. Using this identity, it is also possible to give a direct proof of theorem 2.3, using an induction argument similar to the proof of proposition 1.1.

As in 1.1 , we can specialize theorem 2.3 to obtain the following formula.

Corollary. For a generic complex linear form $z$ on $E$, and for generic complex numbers $y_{1}, \ldots, y_{N}$, the product

$$
\prod_{k=1}^{N} \frac{1}{1-e^{y_{k}+\left\langle z, \alpha_{k}\right\rangle}}
$$

is equal to

$$
\sum_{\sigma \in \mathcal{B}(\Delta)} \sum_{g \in G(\sigma, q)} \prod_{j \in \sigma} \frac{q^{-1} \varepsilon_{\sigma, j}}{1-e^{q^{-1} \varepsilon_{\sigma, j}\left(y_{j}+2 i \pi g_{j}+\left\langle z, \alpha_{j}\right\rangle\right)}} \prod_{k \notin \sigma} \frac{1}{1-e^{y_{k}-\sum_{j \in \sigma} c_{j k}\left(y_{j}+2 i \pi g_{j}\right)}} .
$$


2.4. Fourier transforms and polyhedral cones. Both maps $C \mapsto R(C)$ (defined in 1.5) and $C \mapsto F(C, L)$ may be understood via Fourier transforms of certain generalized functions. We consider a real vector space $W$ of dimension $N$; we use the function notation $\Theta(y)$ for a generalized function $\Theta$ on $W^{*}$, although the value of $\Theta$ at a particular point $y$ may not make any sense. We say that $\Theta$ is smooth on an open subset $U$ of $W^{*}$ if there exists a smooth function $\theta(y)$ on $U$ such that

$$
(\Theta, \varphi d y)=\int_{W^{*}} \theta(y) \varphi(y) d y
$$

for all test functions $\varphi$ with compact support contained in $U$.

For $f \in \mathcal{C}(W)$, the formula

$$
\mathcal{F}(f)(y):=\int_{W} e^{-i\langle y, w\rangle} f(w) d w
$$

defines a generalized function $\mathcal{F}(f)$, the Fourier transform of $f$. Remark that the characteristic function $[C]$ of a cone $C$ generated by a basis $\left(w_{1}, \ldots, w_{N}\right)$ of $W$, satisfies

$$
\left(\prod_{k=1}^{N}\left\langle y, w_{k}\right\rangle\right) \mathcal{F}([C])(y)=\operatorname{vol}|w|
$$

so that the generalized function $\mathcal{F}([C])(y)$ is smooth on the complement of the union of hyperplanes $w_{k}=0$, and is equal to $R(C)(i y)$ on this open subset. On the other hand, if a polyhedral cone $C$ contains a line $\ell$, then for any $w \in \ell$, we have

$$
\langle y, w\rangle \mathcal{F}([C])(y)=0
$$

so that $\mathcal{F}([C])$ is identically 0 outside the hyperplane of $W^{*}$ orthogonal to $\ell$. Thus, for any $f \in \mathcal{C}(W)$, we have for generic $y \in W^{*}$

$$
\mathcal{F}(f)(y)=R(f)(i y)
$$

and this determines $R(f)$ uniquely.

Now fix a lattice $L$ in $W$, and denote by $W_{\mathbb{Q}}$ the rational vector space generated by $L$. We can identify polyhedral cones in $W_{\mathbb{Q}}$ with rational polyhedral cones in $W$, and hence $\mathcal{C}\left(W_{\mathbb{Q}}\right)$ identifies with a subgroup of $\mathcal{C}(W)$. For $f \in \mathcal{C}\left(W_{\mathbb{Q}}\right)$, the formula

$$
\mathcal{F}(f, L)(y):=\sum_{l \in L} f(l) e^{i\langle y, l\rangle}
$$

defines a generalized function $\mathcal{F}(f, L)$ on $W^{*}$. If $C=C\left(w_{1}, \ldots, w_{N}\right)$ is a simplicial cone generated by a basis $\left(w_{1}, \ldots, w_{N}\right)$ of $L$, then

$$
\left(\prod_{k=1}^{N}\left(1-e^{i\left\langle y, w_{k}\right\rangle}\right)\right) \mathcal{F}([C], L)(y)=1
$$

so that $\mathcal{F}([C], L)(y)$ coincides with $F(C, L)(i y)$ on the complement of the union of hyperplanes $\left\langle y, w_{k}\right\rangle \in 2 \pi \mathbb{Z}$. On the other hand, if a rational polyhedral cone $C$ contains a line, then it contains a rational line $\ell$, and we have for all $w \in \ell \cap L$

$$
\left(1-e^{i\langle y, w\rangle}\right) \mathcal{F}([C], L)(y)=0 .
$$

Therefore, $\mathcal{F}([C], L)$ is identically 0 on the complement of the union of the hyperplanes $\langle y, w\rangle \in 2 \pi \mathbb{Z}$. We conclude that for any $f \in \mathcal{C}\left(W_{\mathbb{Q}}\right)$, the generalized function $\mathcal{F}(f, L)(y)$ is equal to the function $F(f, L)(i y)$ for $y$ outside a countable union of hyperplanes. 


\section{VECTOR PARTITION FUNCTIONS}

3.1. Chambers and partition polyhedra. We consider a real vector space $W$ with a basis $\left(w_{1}, \ldots, w_{N}\right)$ and a surjective linear map $p: W \rightarrow E$ with kernel $V$ of dimension $n$. We set $p\left(w_{k}\right):=\alpha_{k}$ for $1 \leq k \leq N$, and $\Delta=\left(\alpha_{1}, \ldots, \alpha_{N}\right)$. We denote by $C$ the convex cone in $W$ generated by $w_{1}, \ldots, w_{N}$, and we set $C(\Delta):=p(C)$.

For any subset $\kappa$ of $\Delta$, we denote by $C(\kappa) \subset E$ the convex cone generated by the $\alpha_{j}(j \in \kappa)$. Then $C(\Delta)$ is the union of the simplicial cones $C(\sigma)(\sigma \in \mathcal{B}(\Delta))$. To any $h \in C(\Delta)$, we associate the intersection of all cones $C(\sigma)$ which contain $h$. This defines a subdivision of $C(\Delta)$ into polyhedral cones. The interiors of the maximal cones of this subdivision are called chambers.

For any chamber $\gamma$, we set

$$
\mathcal{B}(\Delta, \gamma):=\{\sigma \in \mathcal{B}(\Delta) \mid \gamma \subset C(\sigma)\} .
$$

Then the intersection of the $C(\sigma)(\sigma \in \mathcal{B}(\Delta, \gamma))$ is the closure $\bar{\gamma}$ of $\gamma$.

A subsequence of $\Delta$ is called generating if it contains a basis. We denote by $\mathcal{G}(\Delta)$ the set of generating subsequences of $\Delta$. For a chamber $\gamma$, we set

$$
\mathcal{G}(\Delta, \gamma):=\{\kappa \in \mathcal{G}(\Delta) \mid \gamma \subset C(\kappa)\}
$$

For $h \in E$, we set

$$
V(h):=p^{-1}(h) \text { and } P_{\Delta}(h):=p^{-1}(h) \cap C .
$$

Then $V(h)$ is an affine space with direction $V=\operatorname{ker}(p)$, and $P_{\Delta}(h)$ is a convex polyhedron in $V(h)$, i.e., the intersection of finitely many closed half-spaces. Observe that $P_{\Delta}(h)$ contains no affine line. Moreover, $P_{\Delta}(h)$ is non-empty if and only if $h$ is in $C(\Delta)$. The family $\left(P_{\Delta}(h)\right)_{h \in C(\Delta)}$ is called the family of partition polyhedra defined by $p: W \rightarrow E$ and $C$. This construction is well known, see e.g. [18].

Recall that the recession cone of a convex polyhedron $P \subset W$ is

$$
r e(P):=\{\xi \in W \mid x+\xi \in P \forall x \in P\} .
$$

Clearly, re $\left(P_{\Delta}(h)\right)$ is equal to $P_{\Delta}(0)=C \cap V$, i.e., to the cone of non-negative linear relations between elements of $\Delta$. In particular, the polyhedron $P_{\Delta}(h)$ is bounded if and only if the cone $C(\Delta)$ is acute.

In fact, the structure of $P_{\Delta}(h)$ depends only on the position of $h$ with respect to the decomposition into chambers. To see this, we describe the vertices of $P_{\Delta}(h)$ and their "tangent cones", defined as follows: For any convex polyhedron $P$ in $W$, and for any $x \in P$, the tangent cone of $P$ at $x$ is the closed convex cone generated by $-x+P$; we denote by $C_{x} P$ this (polyhedral) cone. Because $P_{\Delta}(h)$ contains no line, it is uniquely determined by its vertices and the corresponding tangent cones.

For $\sigma \in \mathcal{B}(\Delta)$, we denote by $v_{\sigma}: E \rightarrow W$ the linear map such that $v_{\sigma}\left(\alpha_{j}\right)=w_{j}$ for all $j \in \sigma$. Then $v_{\sigma}\left(\alpha_{k}\right)=\sum_{j \in \sigma} c_{j k} w_{j}$ for all $k \notin \sigma$. Moreover, $\rho_{\sigma}(w)=$ $w-v_{\sigma} p(w)$ for all $w \in W$. If $\gamma$ is a chamber, and $\sigma \in \mathcal{B}(\Delta, \gamma)$, then

$$
P_{\Delta}(h) \cap \bigoplus_{j \in \sigma} \mathbb{R} w_{j}=\left\{v_{\sigma}(h)\right\}
$$

for all $h \in \bar{\gamma}$. For $\kappa \in \mathcal{G}(\Delta, \gamma)$, we set

$$
P_{\Delta}(h) \cap \bigoplus_{k \in \kappa} \mathbb{R} w_{k}:=F_{\kappa}(h) .
$$

Now we can state the following result, whose proof is a direct check. 
Proposition. Let $\gamma$ be a chamber.

(i) For any $h \in \gamma$, the convex polyhedron $P_{\Delta}(h)$ is simple, with vertices $v_{\sigma}(h)(\sigma \in$ $\mathcal{B}(\Delta, \gamma)$ ) and corresponding tangent cones $\rho_{\sigma}(C)$. More generally, for any $\kappa \in$ $\mathcal{G}(\Delta, \gamma)$, the set $F_{\kappa}(h)$ is a face of dimension $|\kappa|-n$ of $P_{\Delta}(h)$, and this defines a bijection between $\mathcal{G}(\Delta, \gamma)$ and the set of faces of $P_{\Delta}(h)$.

(ii) For any $h \in \bar{\gamma}$, the faces of $P_{\Delta}(h)$ are still the $F_{\kappa}(h)(\kappa \in \mathcal{G}(\Delta, \gamma))$, with possible repetitions. Moreover, the dual of the tangent cone to $P_{\Delta}(h)$ at its vertex $v$ has a simplicial subdivision whose maximal cones are the duals of the cones $\rho_{\sigma}(C)\left(\sigma \in \mathcal{B}(\Delta, \gamma), v=v_{\sigma}(h)\right)$.

3.2. Characteristic functions of partition polyhedra. In this section, we deduce from corollary 1.4 formulae for the characteristic functions of the partition polyhedron $P_{\Delta}(h)$ and of its interior in $V(h)$. Both functions live in the additive group $\mathrm{He}(V(h))$ generated by all characteristic functions of convex polyhedra in $V(h)$. We denote by $L H e(V(h))$ the subgroup of $H e(V(h))$ generated by characteristic functions of convex polyhedra which contain affine lines.

Theorem. Let $h \in C(\Delta)$ and let $\gamma$ be a chamber such that $h \in \bar{\gamma}$.

(i) We have in $\mathrm{He}(V(h))$ :

$$
\left[P_{\Delta}(h)\right]=\sum_{\sigma \in \mathcal{B}(\Delta, \gamma)}\left[v_{\sigma}(h)+\rho_{\sigma}(C)\right]
$$

modulo LHe(V(h)). Furthermore, if $h \in C(\Delta)^{0}$, then

$$
\left[P_{\Delta}(h)^{0}\right]=\sum_{\sigma \in \mathcal{B}(\Delta, \gamma)}\left[v_{\sigma}(h)+\rho_{\sigma}(C)^{0}\right] .
$$

(ii) For any vertex $v$ of $P_{\Delta}(h)$, with tangent cone $C_{v} P_{\Delta}(h)$, we have in $\mathcal{C}(V)$ :

$$
\left[C_{v} P_{\Delta}(h)\right]=\sum_{\sigma \in \mathcal{B}(\Delta, \gamma), v=v_{\sigma}(h)}\left[\rho_{\sigma}(C)\right]
$$

modulo $\mathcal{L} C(V)$. Furthermore, if $h \in C(\Delta)^{0}$, then

$$
\left[C_{v} P_{\Delta}(h)^{0}\right]=\sum_{\sigma \in \mathcal{B}(\Delta, \gamma), v=v_{\sigma}(h)}\left[\rho_{\sigma}(C)^{0}\right]
$$

modulo $\mathcal{L} C(V)$.

Proof. (i) Choose a regular point $x \in C$. Then, restricting identity (1.4.3) to $V(h)$, we obtain

$$
\left[P_{\Delta}(h)\right]-\sum_{\sigma \in \mathcal{B}(\Delta)}\left[V(h) \cap\left(C_{x}^{\prime \sigma}+\rho_{\sigma}(C)\right)\right]=g
$$

for some $g \in \operatorname{LHe}(V(h))$. Now we choose $x$ such that $p(x) \in \gamma$ and that no hyperplane generated by some of the $\alpha_{k}$ separates $h$ and $p(x)$. If $V(h)$ meets $C_{x}^{\prime \sigma}+\rho_{\sigma}(C)$, then $h$ is in $p\left(C_{x}^{\prime \sigma}\right)$, and hence $h=\sum_{j \in \sigma} h_{\sigma, j} \alpha_{j}$ with $h_{\sigma, j}>0$ for all $j \in \sigma_{-}(x)$. By the assumption on $x$, the set $\sigma_{-}(x)$ must be empty, that is, $\sigma \in \mathcal{B}(\Delta, \gamma)$. Therefore, $C_{x}^{\prime \sigma}=C(\sigma)$. Moreover, we have

$$
V(h) \cap\left(C_{x}^{\prime \sigma}+\rho_{\sigma}(C)\right)=v_{\sigma}(h)+\rho_{\sigma}(C) .
$$

Indeed, we have $v_{\sigma}(h) \in C(\sigma)=C_{x}^{\prime \sigma}$ and hence $v_{\sigma}(h)+\rho_{\sigma}(C) \subset V(h) \cap$ $\left(C_{x}^{\prime \sigma}+\rho_{\sigma}(C)\right)$. On the other hand, if $u \in V(h) \cap\left(C_{x}^{\prime \sigma}+\rho_{\sigma}(C)\right)$, then $u-\rho_{\sigma}(u) \in$ 
$C(\sigma)$. But $u-\rho_{\sigma}(u)=v_{\sigma} p(u)=v_{\sigma}(h)$ and hence $u \in v_{\sigma}(h)+\rho_{\sigma}(C)$. This proves $(3.2 .1)$.

Assume now that $h \in C(\Delta)^{0}$. Then $P_{\Delta}(h)$ contains points $\left(x_{1}, \ldots, x_{N}\right)$ such that $x_{k}>0$ for all $k$. It follows that

$$
P_{\Delta}(h)^{0}=C^{0} \cap V(h) .
$$

For $x$ as above, and for small $\varepsilon>0$, set $x^{0}:=h-\varepsilon x$. Then $x^{0}$ is regular. Moreover, identity (1.4.4) implies that

$$
\left[P_{\Delta}(h)^{0}\right]-\sum_{\sigma \in \mathcal{B}(\Delta)}\left[V(h) \cap\left(C_{x^{0}}^{\prime \prime \sigma}+\rho_{\sigma}(C)^{0}\right)\right]=g^{0}
$$

for some $g^{0} \in L H e(V(h))$.

If $V(h)$ meets $C_{x^{0}}^{\prime \prime \sigma}+\rho_{\sigma}(C)^{0}$, then $h \in p\left(C_{x^{0}}^{\prime \prime \sigma}\right)$. If moreover $x \notin C(\sigma)$, then there exists $j \in \sigma$ such that $x_{\sigma, j}<0$. By our choice of $x$, we have $h_{\sigma, j} \leq 0$. Then either $h_{\sigma, j}=0$ and $x_{\sigma, j}^{0}>0$, or $h_{\sigma, j}<0$ and $x_{\sigma, j}^{0}<0$. In both cases, $h \notin p\left(C_{x^{0}}^{\prime \prime \sigma}\right)$, a contradiction. So $x \in C(\sigma)$, that is, $\sigma \in \mathcal{B}(\Delta, \gamma)$. Arguing as above, we then have

$$
V(h) \cap\left(C_{x^{0}}^{\prime \prime \sigma}+\rho_{\sigma}(C)^{0}\right)=v_{\sigma}(h)+\rho_{\sigma}(C)^{0} .
$$

This proves (3.2.2).

(ii) is deduced from (i) by a process of limit at $v$, as follows. For an arbitrary convex polyhedron $P \subset V(h)$, and for $\varepsilon>0$, consider the function $x \mapsto[P](v+\varepsilon x)$. Then the pointwise limit of this function as $\varepsilon \rightarrow 0$ is $\left[C_{v} P\right]$ if $v \in P$, and 0 otherwise.

Assume that $v \in P$. Remark that $\left[C_{v} P\right]$ is in $\mathcal{L} C(V)$ in the case where $P$ contains an affine line (then $P$ contains an affine line through $v$ ) and also in the case where $P$ is an affine cone with vertex $s \neq v$ (then $C_{v} P$ contains the line $\mathbb{R}(s-v)$ ).

Now write

$$
\left[P_{\Delta}(h)\right]=\sum_{\sigma \in \mathcal{B}(\Delta, \gamma)}\left[v_{\sigma}(h)+\rho_{\sigma}(C)\right]+\sum_{j} a_{j}\left[P_{j}\right]
$$

where each $P_{j}$ is a convex polyhedron in $V(h)$ which contains an affine line. Evaluating both sides at $v+\varepsilon x$ and letting $\varepsilon \rightarrow 0$, we obtain

$$
\left[C_{v} P_{\Delta}(h)\right]=\sum_{\sigma \in \mathcal{B}(\Delta, \gamma), v=v_{\sigma}(h)}\left[\rho_{\sigma}(C)\right]+g
$$

with $g \in \mathcal{L} C(V)$. This proves (3.2.3). Similarly, (3.2.4) follows from (3.2.2).

3.3. Fourier transforms of partition polyhedra. In this section, we obtain a closed formula for the Fourier transform of the Lebesgue measure on $P_{\Delta}(h)$. We choose Lebesgue measures on $W$ and $E$; this induces a Lebesgue measure $d s$ on each fiber of $p$. For any basis $\sigma$, we denote by $\mu(\sigma)$ the volume of the convex polytope $\left\{\sum_{j \in \sigma} t_{j} \alpha_{j} \mid 0 \leq t_{j} \leq 1\right\}$. For all $h \in C(\Delta)$ and $y$ in the interior of the dual cone of $C \cap V$, we set

$$
\mathcal{V}_{\Delta}(y, h):=\int_{P_{\Delta}(h)} e^{-\langle y, w\rangle} d s
$$

(this makes sense because $y$ is bounded from below on $P_{\Delta}(h)$ ). In particular, if the cone $C(\Delta)$ is acute, then $\mathcal{V}_{\Delta}(y, h)$ is defined for all $y \in W^{*}$, and $\mathcal{V}_{\Delta}(h):=\mathcal{V}_{\Delta}(0, h)$ is the volume of $P_{\Delta}(h)$. 
Proposition. For any $h \in C(\Delta)$, for any chamber $\gamma$ such that $h \in \bar{\gamma}$, and for generic $y$ in the interior of the dual cone of $C \cap V$, we have

$$
\mathcal{V}_{\Delta}(y, h)=\sum_{\sigma \in \mathcal{B}(\Delta, \gamma)} \frac{e^{-\left\langle y, v_{\sigma}(h)\right\rangle}}{\mu(\sigma) \prod_{k \notin \sigma}\left(y_{k}-\sum_{j \in \sigma} c_{j k} y_{j}\right)} .
$$

Proof. Taking the Fourier transform (see 2.4) of (3.2.1), we obtain for $h \in \bar{\gamma}$ and for generic $y \in W^{*}$ :

$$
\int_{P_{\Delta}(h)} e^{-i\langle y, s\rangle} d s=\sum_{\sigma \in \mathcal{B}(\Delta, \gamma)} \int_{v_{\gamma}(h)+\rho_{\gamma}(C)} e^{-i\langle y, s\rangle} d s .
$$

This gives our relation.

Definition. We set

$$
\mathcal{V}_{\Delta, \gamma}(y, h):=\sum_{\sigma \in \mathcal{B}(\Delta, \gamma)} \frac{e^{-\left\langle y, v_{\sigma}(h)\right\rangle}}{\mu(\sigma) \prod_{k \notin \sigma}\left(y_{k}-\sum_{j \in \sigma} c_{j k} y_{j}\right)} .
$$

This function coincides with $\mathcal{V}_{\Delta}(y, h)$ for $h \in \bar{\gamma}$, and is defined outside a union of finitely many hyperplanes.

Corollary. If the cone $C(\Delta)$ is acute, then we have

$$
\mathcal{V}_{\Delta}(h)=\sum_{\sigma \in \mathcal{B}(\Delta, \gamma)} \frac{\left\langle y, v_{\sigma}(h)\right\rangle^{n}}{n ! \mu(\sigma) \prod_{k \notin \sigma}\left(-y_{k}+\sum_{j \in \sigma} c_{j k} y_{j}\right)}
$$

for all $h \in \bar{\gamma}$, and for generic $y \in W^{*}$. In particular, the function $h \rightarrow \mathcal{V}_{\Delta}(h)=$ $\operatorname{vol} P_{\Delta}(h)$ is a homogeneous polynomial function of degree $n=N-d$ on the closure of each chamber.

Proof. Fix $h \in \bar{\gamma}$ and generic $y \in W^{*}$. Expanding

$$
\mathcal{V}_{\Delta}(t y, h)=\int_{P(h)} e^{-t\langle y, w\rangle} d s
$$

into a power series in $t$ and letting $t \rightarrow 0$, we obtain our formula.

3.4. Vector partition functions and their generalizations. In this section, we obtain a discrete analog of proposition 3.3. We maintain the assumptions of 3.1, and we assume furthermore that $p$ maps the lattice $L=\bigoplus_{k=1}^{N} \mathbb{Z} w_{k} \subset W$ onto a lattice $\Lambda$ in $E$. These lattices define uniquely Lebesgue measures on $W$ and $E$; for $\sigma \in \mathcal{B}(\Delta)$, observe that

$$
\operatorname{vol}\left\{\sum_{j \in \sigma} t_{j} \alpha_{j} \mid 0 \leq t_{j} \leq 1\right\}=\left[\Lambda: \bigoplus_{j \in \sigma} \mathbb{Z} \alpha_{j}\right]
$$

so that the notation $\mu(\sigma)$ of 3.3 is consistent with the notation of 2.2 .

For $\lambda \in C(\Delta) \cap \Lambda$ and for $y$ in the interior of the dual cone of $C \cap V$, we set

$$
\mathcal{P}_{\Delta}(y, \lambda):=\sum_{w \in P_{\Delta}(\lambda) \cap L} e^{-\langle y, w\rangle}=\sum e^{-x_{1}\left\langle y, w_{1}\right\rangle-\cdots-x_{N}\left\langle y, w_{N}\right\rangle}
$$

(sum over all non-negative integers $x_{1}, \ldots, x_{N}$ such that $\sum_{k=1}^{N} x_{k} \alpha_{k}=\lambda$ ). This series converges, because $y$ is bounded from below on $P_{\Delta}(\lambda)$. 
We also set

$$
\mathcal{P}_{\Delta}^{0}(y, \lambda):=\sum_{w \in P_{\Delta}(\lambda)^{0} \cap L} e^{-\langle y, w\rangle}=\sum e^{-x_{1}\left\langle y, w_{1}\right\rangle-\cdots-x_{N}\left\langle y, w_{N}\right\rangle}
$$

(sum over all positive integers $x_{1}, \ldots, x_{N}$ such that $\sum_{k=1}^{N} x_{k} \alpha_{k}=\lambda$ ).

If the cone $C(\Delta)$ is acute, then the functions $\mathcal{P}_{\Delta}(y, \lambda)$ and $\mathcal{P}_{\Delta}^{0}(y, \lambda)$ are defined for all $y \in W^{*}$. In particular, $\mathcal{P}_{\Delta}(\lambda):=\mathcal{P}_{\Delta}(0, \lambda)$ is the number of solutions in nonnegative integers of the equation $\sum_{k=1}^{N} x_{k} \alpha_{k}=\lambda$, i.e., $\mathcal{P}_{\Delta}$ is the vector partition function associated to $\Delta$. We call $\mathcal{P}_{\Delta}^{0}$ the strict vector partition function.

Theorem. Let $\lambda \in C(\Delta) \cap \Lambda$; let $\gamma$ be a chamber such that $\lambda \in \bar{\gamma}$. Then, for generic $y$ in the interior of the dual cone of $C \cap V$, we have

$$
\mathcal{P}_{\Delta}(y, \lambda)=\sum_{\sigma \in \mathcal{B}(\Delta, \gamma)} \frac{e^{-\left\langle y, v_{\sigma}(\lambda)\right\rangle}}{\mu(\sigma)} \sum_{g \in G(\sigma)} \frac{e^{2 i \pi \lambda}(g)}{\prod_{k \notin \sigma}\left(1-e^{-2 i \pi \alpha_{k}}(g) e^{-y_{k}+\sum_{j \in \sigma} c_{j k} y_{j}}\right)} .
$$

Furthermore, if $\lambda \in C(\Delta)^{0}$, then

$$
\mathcal{P}_{\Delta}^{0}(y, \lambda)=(-1)^{n} \sum_{\sigma \in \mathcal{B}(\Delta, \gamma)} \frac{e^{-\left\langle y, v_{\sigma}(\lambda)\right\rangle}}{\mu(\sigma)} \sum_{g \in G(\sigma)} \frac{e^{2 i \pi \lambda}(g)}{\prod_{k \notin \sigma}\left(1-e^{2 i \pi \alpha_{k}}(g) e^{y_{k}-\sum_{j \in \sigma} c_{j k} y_{j}}\right)} .
$$

Proof. Consider the formal power series

$$
f\left(P_{\Delta}(\lambda), L\right):=\sum_{w \in P_{\Delta}(\lambda) \cap L} e^{w} .
$$

We show that this series is summable (see 2.1) with sum

$$
\sum_{\sigma \in \mathcal{B}(\Delta, \gamma)} \frac{e^{v_{\sigma}(\lambda)}}{\mu(\sigma)} \sum_{g \in G(\sigma)} \frac{e^{2 i \pi \lambda}(g)}{\prod_{k \notin \sigma}\left(1-e^{-2 i \pi \alpha_{k}}(g) e^{w_{k}-\sum_{j \in \sigma} c_{j k} w_{j}}\right)} .
$$

This implies (3.4.1).

For $\sigma \in \mathcal{B}(\Delta, \gamma)$, set

$$
f\left(v_{\sigma}(\lambda)+\rho_{\sigma}(C), L\right):=\sum_{w \in\left(v_{\sigma}(\lambda)+\rho_{\sigma}(C)\right) \cap L} e^{w} .
$$

Then, using 3.2 and 2.1, we see that

$$
f\left(P_{\Delta}(\lambda), L\right)-\sum_{\sigma \in \mathcal{B}(\Delta, \gamma)} f\left(v_{\sigma}(\lambda)+\rho_{\sigma}(C), L\right)
$$

is summable with sum 0 . Moreover, setting

$$
M_{\sigma}:=L_{\sigma} \oplus \rho_{\sigma}(L)=L_{\sigma}+L
$$

we have: $v_{\sigma}(\lambda) \in M_{\sigma}$ and

$$
f\left(v_{\sigma}(\lambda)+\rho_{\sigma}(C), M_{\sigma}\right)=\frac{e^{v_{\sigma}(\lambda)}}{\prod_{k \notin \sigma}\left(1-e^{\rho_{\sigma}\left(w_{k}\right)}\right)}=\frac{e^{v_{\sigma}(\lambda)}}{\prod_{k \notin \sigma}\left(1-e^{\left.w_{k}-\sum_{j \in \sigma} c_{j k} w_{j}\right)}\right)} .
$$


As in the proof of theorem 2.2, it follows that $f\left(v_{\sigma}(\lambda)+\rho_{\sigma}(C), L\right)$ is summable with sum

$$
\frac{e^{v_{\sigma}(\lambda)}}{\mu(\sigma)} \sum_{g \in G(\sigma)} \frac{e^{2 i \pi \lambda}(g)}{\prod_{k \notin \sigma}\left(1-e^{-2 i \pi \alpha_{k}}(g) e^{w_{k}-\sum_{j \in \sigma} c_{j k} w_{j}}\right)} .
$$

This proves (3.4.1).

For (3.4.2), we deduce from theorem 3.2 that the series

$$
f\left(P_{\Delta}(\lambda)^{0}, L\right)-\sum_{\sigma \in \mathcal{B}(\Delta, \gamma)} f\left(v_{\sigma}(\lambda)+\rho_{\sigma}(C)^{0}, L\right)
$$

is summable with sum 0 . Moreover, each series $f\left(v_{\sigma}(\lambda)+\rho_{\sigma}(C)^{0}, M_{\sigma}\right)$ is summable with sum

$$
e^{v_{\sigma}(\lambda)} \prod_{k \notin \sigma} \frac{e^{\rho_{\sigma}(\lambda)}}{1-e^{\rho_{\sigma}(\lambda)}}=(-1)^{n} e^{v_{\sigma}(\lambda)} \prod_{k \notin \sigma} \frac{1}{1-e^{-\rho_{\sigma}\left(w_{k}\right)}} .
$$

It follows that $f\left(v_{\sigma}+\rho_{\sigma}(C)^{0}, L\right)$ is summable with sum

$$
\frac{(-1)^{n} e^{v_{\sigma}(\lambda)}}{\mu(\sigma)} \sum_{g \in G(\sigma)} \frac{e^{2 i \pi \lambda}(g)}{\prod_{k \notin \sigma}\left(1-e^{2 i \pi \alpha_{k}}(g) e^{-w_{k}+\sum_{j \in \sigma} c_{j k} w_{j}}\right)} .
$$

3.5. Euler-MacLaurin formula for vector partition functions. In this section, we express $\mathcal{P}_{\Delta}(y, \lambda)$ and $\mathcal{P}_{\Delta}^{0}(y, \lambda)$ (defined in 3.4) in terms of the values at $\lambda$ of the function $h \mapsto \mathcal{V}_{\Delta}(y, h)$ and of its partial derivatives. For this, we introduce series of differential operators with constant coefficients called Todd operators, as follows.

For any complex number $a$, we set

$$
\operatorname{Todd}(a, z):=\frac{z}{1-a e^{-z}}=\sum_{m=0}^{\infty} c(a, m) z^{m} .
$$

Then $\operatorname{Todd}(a, z)$ is an analytic function of $z$ for small $z$. In particular, we have

$$
\operatorname{Todd}(1, z)=\frac{z}{1-e^{-z}}=1+\frac{1}{2} z+\sum_{m=1}^{\infty}(-1)^{m-1} \frac{B_{m}}{(2 m) !} z^{2 m}
$$

where the $B_{m}$ 's are the Bernoulli numbers.

For $u \in E$ and $\varphi$ a smooth function on $E$, we denote by $\partial(u) \varphi$ the derivative of $\varphi$ in the direction $u$. We define the Todd operator $\operatorname{Todd}(a, \partial(u))$ by the formula

$$
\operatorname{Todd}(a, \partial(u)):=\frac{\partial(u)}{1-a e^{-\partial(u)}}=\sum_{m=0}^{\infty} c(a, m) \partial(u)^{m} .
$$

Then $\operatorname{Todd}(a, \partial(u))$ is a differential operator of infinite order, with constant coefficients. It acts for example in the space of polynomial functions on $E$. For $y \in \mathbb{R}$, we define $\operatorname{Todd}(a, \partial(u)+y)$ in a similar way. Finally, for $g \in E^{*} / \Lambda^{*}$ and $y \in W^{*}$, we set

$$
\operatorname{Todd}_{\Delta}(g, \partial+y):=\prod_{k=1}^{N} \operatorname{Todd}\left(e^{-2 i \pi \alpha_{k}}(g), \partial\left(\alpha_{k}\right)+y_{k}\right)
$$


with $y_{k}=\left\langle y, w_{k}\right\rangle$. Then $\operatorname{Todd}_{\Delta}(g, \partial+y)$ is a series of differential operators with constant coefficients, and $\operatorname{Todd}_{\Delta}(g, \partial)$ is a differential operator of infinite order with constant coefficients.

Theorem. Let $\gamma$ be a chamber, and let $\mathcal{V}_{\Delta, \gamma}(y, h)$ be as in 3.3.

(i) The series

$$
\operatorname{Todd}_{\Delta}(g, \partial+y) \mathcal{V}_{\Delta, \gamma}(y, h)
$$

converges for all $g \in E^{*} / \Lambda^{*}$, and for small generic $y \in W^{*}$. Moreover, the sum of this series vanishes if $g$ is not in the finite set

$$
G(\gamma):=\bigcup_{\sigma \in \mathcal{B}(\Delta, \gamma)} G(\sigma) .
$$

(ii) For $\lambda \in \bar{\gamma} \cap \Lambda$ and for small generic $y$ in the interior of the dual cone of $C \cap V$, we have:

$$
\mathcal{P}_{\Delta}(y, \lambda)=\left.\sum_{g \in G(\gamma)} e^{2 i \pi \lambda}(g) \operatorname{Todd}_{\Delta}(g, \partial+y) \mathcal{V}_{\Delta, \gamma}(y, h)\right|_{h=\lambda} .
$$

Furthermore, if $\lambda \in C(\Delta)^{0}$, then

$$
\mathcal{P}_{\Delta}^{0}(y, \lambda)=\left.\sum_{g \in G(\gamma)} e^{2 i \pi \lambda}(g) \operatorname{Todd}_{\Delta}(-g,-\partial-y) \mathcal{V}_{\Delta, \gamma}(y, h)\right|_{h=\lambda} .
$$

Proof. (i) Using proposition 3.3, it is enough to check the assertions for the series

$$
\operatorname{Todd}_{\Delta}(g, \partial+y) e^{-\left\langle y, v_{\sigma}(h)\right\rangle}
$$

for $\sigma \in \mathcal{B}(\Delta, \gamma)$. Observe that

$$
\left(\partial\left(\alpha_{k}\right)+y_{k}\right) e^{-\left\langle y, v_{\sigma}(h)\right\rangle}=\left(y_{k}-\left\langle y, v_{\sigma}\left(\alpha_{k}\right)\right\rangle\right) e^{-\left\langle y, v_{\sigma}(h)\right\rangle}
$$

and that, for all $k \in \sigma$ :

$$
y_{k}-\left\langle y, v_{\sigma}\left(\alpha_{k}\right)\right\rangle=0 \text {. }
$$

Moreover, the series

$$
\operatorname{Todd}\left(e^{-2 i \pi \alpha_{k}}(g), \partial\left(\alpha_{k}\right)+y_{k}\right)
$$

has a non-zero constant term if and only if $e^{2 i \pi \alpha_{k}}(g)=1$, and then this constant term is equal to 1 . It follows that

$$
\begin{aligned}
& \operatorname{Todd}_{\Delta}(g, \partial+y) e^{-\left\langle y, v_{\sigma}(h)\right\rangle} \\
& \quad=e^{-\left\langle y, v_{\sigma}(h)\right\rangle} \prod_{k \notin \sigma} \sum_{m=0}^{\infty} c\left(e^{-2 i \pi \alpha_{k}}(g), m\right)\left(y_{k}-\left\langle y, v_{\sigma}\left(\alpha_{k}\right)\right\rangle\right)^{m}
\end{aligned}
$$

if $y \in G(\sigma)$; otherwise, $\operatorname{Todd}_{\Delta}(g, \partial+y) e^{-\left\langle y, v_{\sigma}(h)\right\rangle}=0$.

(ii) We can rewrite the formulae above as

$$
\operatorname{Todd}_{\Delta}(g, \partial+y) e^{-\left\langle y, v_{\sigma}(h)\right\rangle}=e^{-\left\langle y, v_{\sigma}(h)\right\rangle} \prod_{k \notin \sigma} \frac{y_{k}-\sum_{j \in \sigma} c_{j k} y_{j}}{1-e^{-2 i \pi \alpha_{k}}(g) e^{-y_{k}+\sum_{j \in \sigma} c_{j k} y_{j}}}
$$

if $g \in G(\sigma)$; otherwise, $\operatorname{Todd}_{\Delta}(g, \partial+y) e^{-\left\langle y, v_{\sigma}(h)\right\rangle}=0$. Using 3.3 and (3.4.1), we obtain formula (3.5.1). The proof of (3.5.2) is similar. 
Corollary 1. If the cone $C(\Delta)$ is acute, then we have for any $\lambda \in C(\Delta) \cap \Lambda$ and for any chamber $\gamma$ such that $\lambda \in \bar{\gamma}$ :

$$
\mathcal{P}_{\Delta}(\lambda)=\left.\sum_{g \in G(\gamma)} e^{2 i \pi \lambda}(g) \operatorname{Todd}_{\Delta}(g, \partial) \mathcal{V}_{\Delta, \gamma}(h)\right|_{h=\lambda} .
$$

Furthermore, if $\lambda \in C(\Delta)^{0}$, then

$$
\mathcal{P}_{\Delta}^{0}(\lambda)=\left.\sum_{g \in G(\gamma)} e^{2 i \pi \lambda}(g) \operatorname{Todd}_{\Delta}(-g,-\partial) \mathcal{V}_{\Delta, \gamma}(h)\right|_{h=\lambda} .
$$

Definitions. A function $f$ on $\Lambda$ is called

polynomial if $f$ extends to a polynomial function on $E$,

periodic if $f$ factors through $\Lambda / L$ where $L \subset \Lambda$ is a sublattice (then $L$ is a period group for $f$ ),

periodic polynomial if $f$ belongs to the ring generated by polynomial and by periodic functions (then a period group of $f$ is a sublattice $L$ such that $f$ restricts to a polynomial function on each coset of $L$ ) .

The closed formulae above show that the function $\mathcal{P}_{\Delta}\left(\right.$ resp. $\left.\mathcal{P}_{\Delta}^{0}\right)$ is periodic polynomial on the closure of each chamber in $C(\Delta)$ (resp. in $\left.C(\Delta)^{0}\right)$. More precisely, for any $g \in G(\gamma)$, define a polynomial function $\mathcal{P}_{\Delta, g, \gamma}$ on $\Lambda$ by

$$
\mathcal{P}_{\Delta, g, \gamma}:=\operatorname{Todd}_{\Delta}(g, \partial) \mathcal{V}_{\Delta, \gamma} .
$$

Then the function

$$
\lambda \mapsto \mathcal{P}_{\Delta, \gamma}(\lambda):=\sum_{g \in G(\gamma)} e^{2 i \pi \lambda}(g) \mathcal{P}_{\Delta, g, \gamma}(\lambda)
$$

is periodic polynomial on $\Lambda$, and coincides with $\mathcal{P}_{\Delta}$ on $\Lambda \cap \bar{\gamma}$.

Set

$$
\Lambda(\gamma):=\left\{\lambda \in \Lambda \mid e^{2 i \pi \lambda}(g)=1 \forall g \in G(\gamma)\right\} .
$$

Then $\Lambda(\gamma)$ is the intersection of all sublattices $\bigoplus_{j \in \sigma} \mathbb{Z} \alpha_{j}$ for $\sigma \in \mathcal{B}(\Delta, \gamma)$. An element $h \in \Lambda \cap \bar{\gamma}$ belongs to $\Lambda(\gamma)$ if and only if all vertices $v_{\sigma}(h)$ of the polytope $P_{\Delta}(h)$ are integral. According to Ehrhart [10], restriction of $\mathcal{P}_{\Delta, \gamma}$ to $\Lambda(\gamma)$ is polynomial. The following corollary gives a closed formula for this polynomial function.

Corollary 2. The sublattice $\Lambda(\gamma)$ is a period group for $\mathcal{P}_{\Delta, \gamma}$, and restriction of this function to this sublattice coincides with restriction of the polynomial function

$$
\mathcal{E}_{\Delta, \gamma}:=\sum_{g \in G(\gamma)} \mathcal{P}_{\Delta, g, \gamma}
$$

3.6. Vector partition functions and volumes of faces. In this section, we assume that the cone $C(\Delta)$ is acute. We obtain formulae for $\mathcal{P}_{\Delta}(\lambda)$ in terms of the faces of the polytope $P_{\Delta}(\lambda)$. In particular, we refine the results of [20].

First we express the series of differential operators $\operatorname{Todd}_{\Delta}(g, \partial+y)$ occurring in our Euler-MacLaurin formula, in terms of the faces of $P_{\Delta}(h)$ for $h \in \gamma$. Recall that these faces are indexed by the set $\mathcal{G}(\Delta, \gamma)$, see 3.1 .

Observe that $G(\gamma)$ is the union of its subsets

$$
G(\sigma)=\left\{g \in E^{*} / \Lambda^{*} \mid e^{2 i \pi \alpha_{k}}(g)=1 \forall k \in \sigma\right\} .
$$


It follows that $G(\gamma)$ is the disjoint union of its subsets

$$
G(\kappa)^{0}:=\left\{g \in E^{*} / \Lambda^{*} \mid e^{2 i \pi \alpha_{k}}(g)=1 \Leftrightarrow k \in \kappa\right\}
$$

where $\kappa$ runs over $\mathcal{G}(\Delta, \gamma)$. For such a $\kappa$ and for $g \in G(\kappa)^{0}$, we set

$$
R_{\Delta}(g, \partial+y):=\prod_{k \in \kappa} \frac{\partial\left(\alpha_{k}\right)+y_{k}}{1-e^{-\partial\left(\alpha_{k}\right)-y_{k}}} \prod_{k \notin \kappa} \frac{1}{1-e^{-2 i \pi \alpha_{k}}(g) e^{-\partial\left(\alpha_{k}\right)-y_{k}}},
$$

a series of differential operators with constant coefficients. The constant term of the differential operator $R_{\Delta}(g, \partial)$ is $\prod_{k \notin \kappa} \frac{1}{1-e^{-2 i \pi \alpha_{k}}(g)}$. We also set

$$
\Pi_{\kappa}(\partial+y):=\prod_{k \notin \kappa}\left(\partial\left(\alpha_{k}\right)+y_{k}\right)
$$

Then, for all $g \in G(\kappa)^{0}$, we have

$$
\operatorname{Todd}_{\Delta}(g, \partial+y)=R_{\Delta}(g, \partial+y) \Pi_{\kappa}(\partial+y) .
$$

We apply this formula to the function $\mathcal{V}_{\Delta, \gamma}$. Recall that $\mathcal{V}_{\Delta, \gamma}(h)$ is the volume of $P_{\Delta}(h)$ for $h \in \gamma$. More generally, for $h \in \gamma$ and $\kappa \in \mathcal{G}(\Delta, \gamma)$, the volume of the face $F_{\kappa}(h)$ is $\mathcal{V}_{\kappa, \gamma}(h)$ where we still denote by $\gamma$ the chamber (for the system $\kappa$ ) which contains $h$. Using (3.5.3) and (3.5.4), we obtain the following

Proposition. The function $\mathcal{V}_{\Delta, \gamma}$ satisfies the system of partial differential equations

$$
\Pi_{\kappa}(y+\partial) \mathcal{V}_{\Delta, \gamma}(y, h)= \begin{cases}\mathcal{V}_{\kappa, \gamma}(y, h) & \text { if } \kappa \in \mathcal{G}(\Delta, \gamma) \\ 0 & \text { otherwise }\end{cases}
$$

In particular,

$$
\Pi_{\kappa}(\partial) \mathcal{V}_{\Delta, \gamma}= \begin{cases}\mathcal{V}_{\kappa, \gamma} & \text { if } \kappa \in \mathcal{G}(\Delta, \gamma) \\ 0 & \text { otherwise. }\end{cases}
$$

These equations can be used to compute inductively $\mathcal{V}_{\Delta, \gamma}$; indeed, $\mathcal{V}_{\sigma, \gamma}$ is the constant function $\mu(\sigma)^{-1}$ for all $\sigma \in \mathcal{B}(\Delta, \gamma)$. Moreover, we can restate Corollary 3.5.1 as

$$
\mathcal{P}_{\Delta, \gamma}=\sum_{\kappa \in \mathcal{G}(\Delta, \gamma)} \mathcal{P}_{\Delta, \kappa}
$$

where

$$
\mathcal{P}_{\Delta, \kappa}(\lambda):=\left.\sum_{g \in G(\kappa)^{0}} e^{2 i \pi \lambda}(g) R_{\Delta}(g, \partial) \mathcal{V}_{\kappa, \gamma}(h)\right|_{h=\lambda}
$$

is periodic polynomial of degree at most $|\kappa|-n$, and with period group $\sum_{k \in \kappa} \mathbb{Z} \alpha_{k}$. This decomposition of $\mathcal{P}_{\Delta, \gamma}$ is canonical.

Consider now the ring $\mathbb{C}[\partial]$ of differential operators on $E$ with constant coefficients. Let $\mathcal{I}(\Delta, \gamma)$ be the ideal generated by the operators $\Pi_{\kappa}(\partial)$ for $\kappa$ not in $\mathcal{G}(\Delta, \gamma)$. We claim that for any element $D \in \mathbb{C}[\partial]$, there exist constants $c_{\kappa}$ $(\kappa \in \mathcal{G}(\Delta, \gamma))$ such that

$$
D \equiv \sum_{\kappa \in \mathcal{G}(\Delta, \gamma)} c_{\kappa} \Pi_{\kappa}(\partial)(\bmod \mathcal{I}(\Delta, \gamma))
$$


and therefore, by the proposition:

$$
D \mathcal{V}_{\Delta, \gamma}=\sum_{\kappa \in \mathcal{G}(\Delta, \gamma)} c_{\kappa} \mathcal{V}_{\kappa, \gamma}
$$

In other words, the $\mathbb{C}[\partial]$-module generated by the volume function of $P_{\Delta}(h)$ is the vector space spanned by volumes of faces.

Indeed, the ring $\mathbb{C}[\partial]$ is generated by the $\partial\left(\alpha_{k}\right), 1 \leq k \leq N$. Consider a monomial $\prod_{k=1}^{N} \partial\left(\alpha_{k}\right)^{n_{k}}$ and denote by $\nu$ the sequence of all $k$ such that $n_{k}=0$. If $\nu$ is not generating, then the monomial is in $\mathcal{I}(\Delta, \gamma)$. If $\nu$ is generating, then we can express $\alpha_{k}(k \notin \nu)$ as a linear combination of the $\alpha_{j}(j \in \nu)$. Thus, we can write each operator, modulo the ideal $\mathcal{I}(\Delta, \gamma)$, as a linear combination of the $\Pi_{\nu}(\partial)$ and finally we keep only those where $\nu$ is generating and in $\mathcal{G}(\Delta, \gamma)$.

Let $\nu \in \mathcal{G}(\Delta, \gamma)$ and let $g \in G(\nu)^{0}$. Applying the claim to the operator $R_{\Delta}(g, \partial)$ and to the system $\nu$, we see that there exist constants $c_{\kappa}(g)(\kappa \in \mathcal{G}(\Delta, \nu))$ such that

$$
R_{\Delta}(g, \partial) \equiv \sum_{\kappa} c_{\kappa}(g) \Pi_{\kappa}(\partial)(\bmod I(\nu, \gamma))
$$

and therefore

$$
\mathcal{P}_{\Delta, g, \gamma}=R_{\Delta}(g, \partial) \Pi_{\nu}(\partial) \mathcal{V}_{\Delta, \gamma}=R_{\Delta}(g, \partial) \mathcal{V}_{\nu, \gamma}=\sum_{\kappa \in \mathcal{G}(\nu, \gamma)} c_{\kappa}(g) \mathcal{V}_{\kappa, \gamma}
$$

So we obtain the following

Theorem. We have

$$
\mathcal{P}_{\Delta, \gamma}=\sum_{\kappa \in \mathcal{G}(\Delta, \gamma)} \Theta_{\kappa} \mathcal{V}_{\kappa, \gamma}
$$

where

$$
\Theta_{\kappa}(\lambda)=\sum_{g \in G(\kappa)} c_{\kappa}(g) e^{2 i \pi \lambda}(g),
$$

a function on $\Lambda / \sum_{k \in \kappa} \mathbb{Z} \alpha_{k}$.

This expresses the number of points in the rational convex polytope $P_{\Delta}(\lambda)$ in terms of the volumes of its faces and of the periodic functions $\Theta_{\kappa}$; observe that each $\Theta_{\kappa}$ is constant on the sublattice $\Lambda(\gamma)$, with value $\sum_{g \in G(\kappa)} c_{\kappa}(g)$. The resulting formula is not canonical, because the polynomials $\mathcal{V}_{\kappa}$ satisfy linear relations.

The following corollary is a refinement of the result in [20]. It expresses the difference between the periodic polynomial $\mathcal{P}_{\Delta, \gamma}$ and the Ehrhart polynomial $\mathcal{E}_{\Delta, \gamma}$; both functions coincide on $\Lambda(\gamma)$. For $\lambda \in \Lambda$ and $\kappa \in \mathcal{G}(\Delta, \gamma)$ we denote by $[\lambda]_{\kappa}$ the image of $\lambda$ in $\Lambda / \sum_{k \in \kappa} \mathbb{Z} \alpha_{k}$.

Corollary. For each chamber, we have

$$
\mathcal{P}_{\Delta, \gamma}(\lambda)-\mathcal{E}_{\Delta, \gamma}(\lambda)=\sum_{\kappa \in \mathcal{G}(\Delta, \gamma), \kappa \neq \Delta}\left(\Theta_{\kappa}\left([\lambda]_{\kappa}\right)-\Theta_{\kappa}(0)\right) \mathcal{V}_{\kappa, \gamma}(\lambda) .
$$


3.7. Euler-MacLaurin formula and integrals over faces. In this section, we still assume that the cone $C(\Delta)$ is acute. We adapt the approach of 3.6 to express $\mathcal{P}_{\Delta}(y, \lambda)$ in terms of integrals of the function $\mathcal{V}_{\Delta}(y, \lambda)$ and its partial derivatives, over the faces of $P_{\Delta}(\lambda)$.

We identify the ring $\mathbb{C}[\partial]$ with the symmetric algebra $S(E)$, and we consider the overring $S(E \oplus W)=S(E) \otimes S(W)$. Identifying $S(W)$ to the ring of polynomial functions on $W^{*}$, we denote $S(E \oplus W)$ by $\mathbb{C}[\partial, y]$. This ring is graded, and its elements $y_{k}=\left\langle w_{k}, y\right\rangle$ have degree 1 . We denote by $\mathcal{S}(\Delta)$ the graded subring of $\mathbb{C}[\partial, y]$ generated by the $\partial\left(\alpha_{k}\right)+y_{k}$ for $1 \leq k \leq N$, and by the subring $S(V)$ of $S(W)$ (identified to the space of polynomial functions on $W^{*}$ which are invariant under translation by $\left.E^{*}\right)$.

Let $\mathcal{I}(\Delta, \gamma, y)$ be the ideal of $\mathcal{S}(\Delta)$ generated by the operators $\Pi_{\kappa}(\partial+y)$ for $\kappa \notin \mathcal{G}(\Delta, \gamma)$. By proposition 3.6, the function $\mathcal{V}_{\Delta}(y, h)$ is killed by $\mathcal{I}(\Delta, \gamma, y)$. The ring $\mathcal{S}(\Delta)$ with its ideal $\mathcal{I}(\Delta, \gamma, y)$ is considered in [8].

We claim that for any $D \in \mathcal{S}(\Delta)$, there exist $c_{\kappa}(y) \in S(V)(\kappa \in \mathcal{G}(\Delta, \gamma))$ such that

$$
D \equiv \sum_{\kappa} c_{\kappa}(y) \Pi_{\kappa}(\partial+y)(\bmod \mathcal{I}(\Delta, \gamma, y)) .
$$

The proof of this claim is the same as in 3.6 ; for a generating sequence $\nu$ and $k \notin \nu$, we use the relation

$$
\partial\left(\alpha_{k}\right)+y_{k}-\sum_{j \in \nu} c_{j k}\left(\partial\left(\alpha_{j}\right)+y_{j}\right)=\left\langle w_{k}-\sum_{j \in \nu} c_{j k} w_{j}, y\right\rangle
$$

where $w_{k}-\sum_{j \in \nu} c_{j k} w_{j} \in V$, that is, $\alpha_{k}=\sum_{j \in \nu} c_{j k} \alpha_{j}$.

Now, arguing as in 3.6, we obtain for $\nu \in \mathcal{G}(\Delta, \gamma)$ and $g \in G(\nu)^{0}$ :

$$
\operatorname{Todd}_{\Delta}(g, \partial+y) \mathcal{V}_{\nu, \gamma}=\sum_{\kappa \in \mathcal{G}(\nu, \gamma)} c_{\kappa}(g, y) \mathcal{V}_{\kappa, \gamma}
$$

where each $c_{\kappa}(g, y)$ is a series with terms in $S(V)$ which is analytic in a neighborhood of 0 . We can thus consider $c_{\kappa}(g, y)$ as a function on a neighborhood of $E^{*}$ in $W^{*}$. Setting

$$
\Theta_{\kappa}(y, \lambda):=\sum_{g \in G(\kappa)} e^{2 i \pi \lambda}(g) c_{\kappa}(g, y)
$$

we obtain

$$
\mathcal{P}_{\Delta, \gamma}(y, \lambda)=\sum_{\kappa \in \mathcal{G}(\Delta, \gamma)} \Theta_{\kappa}(y, \lambda) \mathcal{V}_{\kappa, \gamma}(y, \lambda) .
$$

For fixed $\lambda$, the series $c_{\kappa}(g, y)$ can be considered as a series of differential operators with constant coefficients on the affine space $V(\lambda)$. We denote by $s$ the variable in this affine space. Then the formula above becomes

$$
\sum_{w \in P_{\Delta}(\lambda) \cap L} e^{-\langle y, w\rangle}=\sum_{\kappa \in \mathcal{G}(\Delta, \gamma)} \int_{F_{\kappa}(\lambda)} \Theta_{\kappa}(-\partial(s), \lambda) e^{-\langle y, s\rangle} d s .
$$

This summation formula holds for the exponential of any small linear form on $W$ and thus for any polynomial function. So we have obtained the following 
Theorem. Let $\gamma$ be a chamber. For each $g \in G(\gamma)$, let $c_{\kappa}(g, y)(\kappa \in \mathcal{G}(\Delta, \gamma))$ be series with terms in $S(V)$ such that

$$
\operatorname{Todd}_{\Delta}(g, \partial+y) \equiv \sum_{\kappa \in \mathcal{G}(\Delta, \gamma)} c_{\kappa}(g, y) \Pi_{\kappa}(\partial+y)(\bmod \mathcal{I}(\Delta, \gamma, y)) .
$$

Then, for any polynomial function $\varphi$ on $W$ and for any $\lambda \in \bar{\gamma}$, we have

$$
\sum_{w \in P_{\Delta}(\lambda) \cap L} \varphi(w)=\sum_{\kappa \in \mathcal{G}(\Delta, \gamma)} \int_{F_{\kappa}(\lambda)} \Theta_{\kappa}(-\partial(s), \lambda) \varphi(s) d s
$$

where

$$
\Theta_{\kappa}(-\partial(s), \lambda)=\sum_{g \in G(\kappa)} e^{2 i \pi \lambda}(g) c_{\kappa}(g,-\partial(s))
$$

is a series of differential operators on $V(\lambda)$ with periodic coefficients of period group $\sum_{k \in \kappa} \mathbb{Z} \alpha_{k}$.

In particular, the function $\lambda \mapsto \sum_{w \in P_{\Delta}(\lambda) \cap L} \varphi(w)$ is periodic polynomial on $\Lambda$, and its restriction to $\Lambda(\gamma)$ is polynomial. Moreover, for fixed $\lambda$, we have expressed the sum of values of any polynomial function at all lattice points of the rational convex polytope $P_{\Delta}(\lambda)$, in terms of integrals over faces of the function and its partial derivatives. This is in the spirit of the Euler-MacLaurin formula of Cappell and Shaneson [8], announced in the case where $P_{\Delta}(\lambda)$ is a simple lattice polytope (that is, $\lambda \in \gamma \cap \Lambda(\gamma)$ ).

\section{LATTICE POINTS IN RATIONAL CONVEX POLYHEDRA}

4.1. Convex polyhedra and partition polyhedra. Let $V$ be a real vector space of dimension $n$. Let $P \subset V$ be a convex polyhedron which contains no line, and with non-empty interior. Let $F_{1}, \ldots, F_{N}$ be the facets of $P$, that is, its faces of codimension one. Write the equation of each $F_{k}$ as

$$
\left\langle u_{k}, v\right\rangle+\lambda_{k}=0
$$

where $u_{k}$ are in $V^{*}, \lambda_{k} \in \mathbb{R}$ and $\left\langle u_{k}, v\right\rangle+\lambda_{k} \geq 0$ for all $v \in P$. Then the $u_{k}$ are "inward pointing normal vectors" to $P$. We have:

$$
P=\left\{v \in V \mid\left\langle u_{k}, v\right\rangle+\lambda_{k} \geq 0(1 \leq k \leq N)\right\} .
$$

Moreover, the recession cone of $P$ (see 3.1$)$ is

$$
r e(P)=\left\{v \in V \mid\left\langle u_{k}, v\right\rangle \geq 0(1 \leq k \leq N)\right\},
$$

the dual cone of $C\left(u_{1}, \ldots, u_{N}\right)$. Since $P$ contains no line, $r e(P)$ is acute, and hence $u_{1}, \ldots, u_{N}$ generate the vector space $V^{*}$.

For $x=\left(x_{1}, \ldots, x_{N}\right) \in \mathbb{R}^{N}$, we set

$$
P(x):=\left\{v \in V \mid\left\langle u_{k}, v\right\rangle+x_{k} \geq 0(1 \leq k \leq N)\right\}
$$

(in particular, $P(\lambda)$ is equal to $P$ ). Then $P(x)$ is a convex polyhedron, obtained from $P$ by parallel motions of its facets; the face structure of $P(x)$ may be very different from that of $P$. 
We will see that the $P(x)$ form a family of partition polyhedra as defined in 3.1; this result is known under various forms, see [18]. We set $W:=\mathbb{R}^{N}$ with canonical basis $\left(w_{1}, \ldots, w_{N}\right)$, and we consider the map

$$
\begin{array}{rlc}
\iota: \quad V & \rightarrow & W \\
v & \mapsto & \left(\left\langle u_{1}, v\right\rangle, \ldots,\left\langle u_{N}, v\right\rangle\right) .
\end{array}
$$

Clearly, $\iota$ is injective; we consider $\iota$ as an inclusion of $V$ into $W$. Denote by $p: W \rightarrow E:=W / V$ the quotient map; set $\alpha_{k}:=p\left(w_{k}\right)$ and $\Delta:=\left(\alpha_{1}, \ldots, \alpha_{N}\right)$. Then we have the following statement, whose straightforward proof is omitted.

Proposition. (i) For any $x \in W$, the affine map

$$
\begin{array}{cccc}
x+\iota: & V & \rightarrow & W \\
v & \mapsto & \left(\left\langle u_{1}, v\right\rangle+x_{1}, \ldots,\left\langle u_{N}, v\right\rangle+x_{N}\right)
\end{array}
$$

induces an isomorphism of $P(x)$ onto $P_{\Delta}(p(x))$.

(ii) A subset $\sigma$ of $\{1, \ldots, N\}$ is a basis of $\Delta$ if and only if the sequence $\left(u_{k}\right)_{k \notin \sigma}$ is a basis of $V^{*}$. In this case, the affine hyperplanes $\left\langle u_{k}, v\right\rangle+x_{k}=0(k \notin \sigma)$ have a unique common point $s_{\sigma}(x)$, and moreover $x+\iota\left(s_{\sigma}(x)\right)=v_{\sigma}(p(x))$.

Observe that $p: W \rightarrow E$ depends only on the set

$$
\mathcal{F}:=\left\{F_{1}, \ldots, F_{N}\right\}
$$

of all facets of $P$. Via $p^{-1}$, the subdivision of $C(\Delta) \subset E$ by closures of chambers defines a subdivision of the cone

$$
C(\mathcal{F}):=p^{-1}(C(\Delta))=\{x \in W \mid P(x) \neq \emptyset\} .
$$

We denote the chambers in $C(\Delta)$ and in $C(\mathcal{F})$ by the same letter $\gamma$, and we use the notation $\mathcal{B}(\mathcal{F}, \gamma)$ for $\mathcal{B}(\Delta, \gamma)$ (the set of bases $\sigma$ of $\Delta$ such that the cone $C(\sigma)$ contains $\gamma$ ).

Combining the proposition above with proposition 3.1, we obtain the following

Corollary. Let $\gamma$ be a chamber in $C(\mathcal{F})$. Then a point $x \in W$ is in $\gamma$ if and only if $P(x)$ is simple with vertices $s_{\sigma}(x)(\sigma \in \mathcal{B}(\mathcal{F}, \gamma))$. In this case, the directions of edges of $P(x)$ at $s_{\sigma}(x)$ are given by the dual vectors of the basis $\left(u_{k}\right)_{k \notin \sigma}$.

4.2. Characteristic functions and Fourier transforms of convex polyhedra. Using the notation and results of 4.1, we can now state theorems 3.2 and 3.3 for arbitrary convex polyhedra which contain no affine line.

Theorem. Let $P \subset V$ be a convex polyhedron of dimension $n$ which contains no line. Let $\mathcal{F}=\left\{F_{1}, \ldots, F_{N}\right\}$ be the set of facets of $P$, with inward pointing normal vectors $u_{1}, \ldots, u_{N}$, and let $\gamma$ be a chamber of $C(\mathcal{F})$. Then

(i) For any $x=\left(x_{1}, \ldots, x_{N}\right) \in \bar{\gamma}$, we have modulo the group generated by characteristic functions of polyhedra which contain lines:

$$
[P(x)]=\sum_{\sigma \in \mathcal{B}(\mathcal{F}, \gamma)}\left[s_{\sigma}(x)+C\left(u_{k, \sigma}^{*}\right)_{k \notin \sigma}\right]
$$

where $\left(u_{k, \sigma}^{*}\right)_{k \notin \sigma}$ is the dual basis of $\left(u_{k}\right)_{k \notin \sigma}$, and where $s_{\sigma}(x)=-\sum_{k \notin \sigma} x_{k} u_{k, \sigma}^{*}$ is the corresponding vertex of $P(x)$. 
(ii) If moreover $d v$ is a Lebesgue measure on $V$, then we have for any generic $u \in C\left(u_{1}, \ldots, u_{N}\right)^{0}$ :

$$
\int_{P(x)} e^{-\langle u, v\rangle} d v=\sum_{\sigma \in \mathcal{B}(\mathcal{F}, \gamma)} e^{-\left\langle u, s_{\sigma}(x)\right\rangle} \frac{\operatorname{vol}\left|u_{k, \sigma}^{*}\right|_{k \notin \sigma}}{\prod_{k \notin \sigma}\left\langle u, u_{k, \sigma}^{*}\right\rangle}
$$

where vol $\left|u_{k, \sigma}^{*}\right|_{k \notin \sigma}$ is the volume of the parallelepiped $\left\{\sum_{k \notin \sigma} t_{k} u_{k, \sigma}^{*} \mid 0 \leq t_{k} \leq 1\right\}$.

Recall (see 1.5) that the Laplace transform of an acute polyhedral cone $C \subset W$ is the rational function $R(C)$ on $W_{\mathbb{C}}^{*}$ such that

$$
R(C)(z)=\int_{C} e^{-\langle w, z\rangle} d w
$$

for $z$ such that $\operatorname{Re}(z)$ is in the interior of the dual cone of $C$. Setting $x=0$ in (4.2.2), we obtain a closed expression for this Laplace transform as a sum of simple fractions.

Corollary 1. Let $C$ be an acute polyhedral cone of dimension $n$. Let $\mathcal{F}$ be the set of all facets of $C$, and let $\gamma$ be a chamber of $C(\mathcal{F})$. Then the Laplace transform of $C$ is given by

$$
R(C)=\sum_{\sigma \in \mathcal{B}(\mathcal{F}, \gamma)} \frac{\operatorname{vol}\left|u_{k, \sigma}^{*}\right|_{k \notin \sigma}}{\prod_{k \notin \sigma} u_{k, \sigma}^{*}} .
$$

Using theorem 3.2, we also obtain the following result.

Corollary 2. Let $P \subset V$ be a convex polyhedron of dimension $n$ which contains no line. Then we have modulo $\mathrm{LHe}(V)$ :

$$
[P]=\sum_{s}\left[s+C_{s} P\right]
$$

(summation over all vertices $s$ of $P$, with tangent cones $C_{s} P$ ). Moreover, for any linear form $u$ on $V$ which is bounded from below on $P$, we have

$$
\int_{P} e^{-\langle u, v\rangle} d v=\sum_{s} e^{-\langle u, s\rangle} R\left(C_{s} P\right) .
$$

In the case where $P$ is bounded, (4.2.4) is due to A. Barvinok (see [2], §2), whereas (4.2.3) is deduced in [3] from the Euler identity on the number of faces of convex polytopes. In fact, the theorem above is an explicit version of corollary 2: the choice of a chamber leads to closed expressions of $\left[C_{s} P\right]$ and of $R\left(C_{s} P\right)$ for all vertices $s$ of $P$. From the geometrical point of view, each choice of a chamber determines an approximation of $P$ by a family of simple polyhedra, and in turn, a subdivision of the dual cone of each $C_{s} P$ into simplicial cones (see proposition 3.1).

4.3. Exponential sums for lattice points in rational convex polyhedra. As in 4.1, we consider a convex polyhedron $P$ in an $n$-dimensional real vector space $V$, such that $P$ is $n$-dimensional and contains no affine line. We assume moreover that $V$ is endowed with a lattice $M$, and that all vertices of $P$ are rational with respect to $M$, i.e., that $P$ is a rational convex polyhedron. We determine the exponential sum

$$
\sum_{v \in P \cap M} e^{-\langle u, v\rangle}
$$


where the linear form $u$ on $V$ is bounded from below on $P$. We use the following notation.

For the equation $\left\langle u_{k}, v\right\rangle+\lambda_{k}=0$ of each facet of $P$, we can assume that $u_{k} \in M^{*}$ (the dual lattice of $M$ ), that $\lambda_{k} \in \mathbb{Z}$ and that $\left(u_{k}, \lambda_{k}\right)$ is primitive in $M^{*} \times \mathbb{Z}$. Together with the assumption that $\left\langle u_{k}, v\right\rangle+\lambda_{k} \geq 0$ on $P$, this determines $\left(u_{k}, \lambda_{k}\right)$ uniquely.

We denote by $T$ the torus $\mathbb{R}^{N} / \mathbb{Z}^{N}$. Then any $\lambda \in \mathbb{Z}^{N}$ defines a character $e^{2 i \pi \lambda}$ of $T$ : For $g \in T$ and a representative $\left(g_{1}, \ldots, g_{N}\right)$ of $g$ in $\mathbb{R}^{N}$, we have

$$
e^{2 i \pi \lambda}(g)=\exp \left(2 i \pi \sum_{k=1}^{N} \lambda_{k} g_{k}\right)
$$

For a basis $\sigma$ of $\mathcal{F}$ (that is, $\left(u_{k}\right)_{k \notin \sigma}$ is a basis of $V^{*}$ ), we denote by $G(\sigma)$ the image in $T=\mathbb{R}^{N} / \mathbb{Z}^{N}$ of the subgroup

$$
\left\{\left(g_{1}, \ldots, g_{N}\right) \in \mathbb{R}^{N} \mid g_{j}=0 \forall j \in \sigma \text { and } \sum_{k \notin \sigma} g_{k} u_{k} \in M^{*}\right\} .
$$

Then $G(\sigma)$ is a finite subgroup of $T$ of order

$$
\mu(\sigma):=\operatorname{vol}\left|u_{k}\right|_{k \notin \sigma}=1 / \operatorname{vol}\left|u_{k, \sigma}^{*}\right|_{k \notin \sigma}
$$

where $\left(u_{k, \sigma}^{*}\right)_{k \notin \sigma}$ is the dual basis to $\left(u_{k}\right)_{k \notin \sigma}$.

Theorem. Let $P=P(\lambda)$ be an $n$-dimensional rational convex polyhedron in $V$, with inward pointing normal vectors $u_{1}, \ldots, u_{N}$, and let $\gamma$ be a chamber such that $\lambda \in \bar{\gamma}$. Then we have for any $u \in C\left(u_{1}, \ldots, u_{N}\right)^{0}$ :

$$
\sum_{v \in P \cap M} e^{-\langle u, v\rangle}=\sum_{\sigma \in \mathcal{B}(\mathcal{F}, \gamma)} \frac{e^{-\left\langle u, s_{\sigma}(\lambda)\right\rangle}}{\mu(\sigma)} \sum_{g \in G(\sigma)} \frac{e^{2 i \pi \lambda}(g)}{\prod_{k \notin \sigma}\left(1-e^{-2 i \pi g_{k}} e^{-\left\langle u, u_{k, \sigma}^{*}\right\rangle}\right)}
$$

and moreover

$$
\sum_{v \in P^{0} \cap M} e^{-\langle u, v\rangle}=(-1)^{n} \sum_{\sigma \in \mathcal{B}(\mathcal{F}, \gamma)} \frac{e^{-\left\langle u, s_{\sigma}(\lambda)\right\rangle}}{\mu(\sigma)} \sum_{g \in G(\sigma)} \frac{e^{2 i \pi \lambda}(g)}{\prod_{k \notin \sigma}\left(1-e^{2 i \pi g_{k}} e^{\left\langle u, u_{k, \sigma}^{*}\right\rangle}\right)} .
$$

Both formulae are proved in the same way as (3.4.1) and (3.4.2). But they are not direct consequences of (3.4.1) and (3.4.2), because the latter apply to the intersection $P \cap \mathbb{Z}^{N}$ where $P \subset V$ is embedded in $\mathbb{R}^{N}$ by $v \mapsto\left(\left\langle u_{1}, v\right\rangle, \ldots,\left\langle u_{N}, v\right\rangle\right)$. Then it may happen that $M$ is strictly contained in $V \cap \mathbb{Z}^{N}$.

Assume now that $P$ is a lattice polyhedron, that is, all vertices of $P$ are in the lattice $M$. Then we obtain using 3.2 :

$$
\sum_{v \in P \cap M} e^{-\langle u, v\rangle}=\sum_{s} e^{-\langle u, s\rangle} F\left(C_{s} P,-u\right)
$$

(sum over all vertices of $P$ ). This formula is due to A. Barvinok, see [2], §4.

4.4. Euler-MacLaurin formula for rational convex polytopes. Let $V$ be a real vector space of dimension $n$, endowed with a lattice $M$. Let $P \subset V$ be a rational convex polytope, i.e., the convex hull of finitely many points of $M_{\mathbb{Q}}$. We assume that the interior $P^{0}$ is not empty. 
For any polynomial function $\varphi: V \rightarrow \mathbb{C}$, we set

$$
i_{P}(\varphi):=\sum_{v \in P \cap M} \varphi(v) \text { and } i_{P^{0}}(\varphi):=\sum_{v \in P^{0} \cap M} \varphi(v) .
$$

We express $i_{P}(\varphi)$ and $i_{P^{0}}(\varphi)$ in terms of the integral of $\varphi$ on $P$ and of the variation of this integral when $P$ is deformed by independent parallel motions of its facets.

We denote by $\mathcal{F}=\left\{F_{1}, \ldots, F_{N}\right\}$ the set of facets of $P$, with equations $\left\langle u_{k}, v\right\rangle+$ $\lambda_{k}=0$ normalized as in 4.3. For $x=\left(x_{1}, \ldots, x_{N}\right) \in \mathbb{R}^{N}$, we denote by $P(x)$ the convex polytope in $V$ defined by the inequalities $\left\langle u_{k}, v\right\rangle+x_{k} \geq 0$ for $1 \leq k \leq N$. In particular, we have $P=P(\lambda)$ where $\lambda=\left(\lambda_{1}, \ldots, \lambda_{N}\right)$. We set:

$$
I_{P}(\varphi)(x):=\int_{P(x)} \varphi(v) d v
$$

where the Lebesgue measure $d v$ on $V$ is normalized so that $V / M$ has volume 1 .

For each basis $\sigma$ of $\mathcal{F}$, we have a finite subgroup $G(\sigma)$ of the torus $T=\mathbb{R}^{N} / \mathbb{Z}^{N}$, see 4.3. For any chamber $\gamma$, we denote by $G(\gamma)$ the union of all $G(\sigma)$ for $\sigma \in \mathcal{B}(\mathcal{F}, \gamma)$. Finally, we set for $g \in T$ :

$$
\operatorname{Todd}_{\mathcal{F}}(g, \partial / \partial x):=\prod_{k=1}^{N} \frac{\partial / \partial x_{k}}{1-e^{-2 i \pi g_{k}} e^{-\partial / \partial x_{k}}} .
$$

Then, arguing as in 3.5 , we obtain the following

Theorem. Let $P=P(\lambda)$ be an $n$-dimensional rational convex polytope in $V$. Let $\varphi: V \rightarrow \mathbb{C}$ be a polynomial function; let $\gamma$ be a chamber such that $\lambda \in \bar{\gamma}$. $\mathbb{R}^{N}$.

(i) The function $x \in \gamma \rightarrow I_{P}(\varphi)(x)$ extends to a polynomial function $I_{P, \gamma}(\varphi)$ on

(ii) For all $g \in T$ such that $g \notin G(\gamma)$, we have

$$
\operatorname{Todd}_{\mathcal{F}}(g, \partial / \partial x) I_{P, \gamma}(\varphi)=0 .
$$

(iii) We have

$$
i_{P}(\varphi)=\left.\sum_{g \in G(\gamma)} e^{2 i \pi \lambda}(g)\left(\operatorname{Todd}_{\mathcal{F}}(g, \partial / \partial x) I_{P, \gamma}(\varphi)(x)\right)\right|_{x=\lambda}
$$

and moreover

$$
i_{P^{0}}(\varphi)=\left.\sum_{g \in G(\gamma)} e^{2 i \pi \lambda}(g)\left(\operatorname{Todd}_{\mathcal{F}}(-g,-\partial / \partial x) I_{P, \gamma}(\varphi)(x)\right)\right|_{x=\lambda} .
$$

Consider now the case where $P$ is a lattice polytope, i.e., all vertices of $P$ are in the lattice $M$. By 4.1, these vertices are the points $s_{\sigma}(\lambda)=-\sum_{k \notin \sigma} \lambda_{k} u_{k, \sigma}^{*}$ for $\sigma \in \mathcal{B}(\mathcal{F}, \gamma)$. Then, by the definition of $G(\sigma)$, we have $e^{2 i \pi \lambda}(g)=1$ for all $g \in G(\sigma)$. So our Euler-MacLaurin formula simplifies as follows.

Corollary. Let $P=P(\lambda)$ be a convex lattice polytope in $V$, let $\varphi$ be a polynomial function on $V$, and let $\gamma$ be a chamber such that $\lambda \in \bar{\gamma}$. Then

$$
i_{P}(\varphi)=\left.\sum_{g \in G(\gamma)}\left(\operatorname{Todd}_{\mathcal{F}}(g, \partial / \partial x) I_{P, \gamma}(\varphi)(x)\right)\right|_{x=\lambda}
$$

and moreover

$$
i_{P^{0}}(\varphi)=\left.\sum_{g \in G(\gamma)}\left(\operatorname{Todd}_{\mathcal{F}}(-g,-\partial / \partial x) I_{P, \gamma}(\varphi)(x)\right)\right|_{x=\lambda} .
$$




\section{REFERENCES}

[1] M. F. Atiyah, Elliptic operators and compact groups, Springer-Verlag, New York, 1974. MR 58:2910

[2] A. I. Barvinok, Computing the volume, counting integral points, and exponential sums, Discrete Comput. Geom. 10 (1993), 123-141. MR 94d:52005

[3] M. Brion and M. Vergne, Lattice points in simple polytopes, J. Amer. Math. Soc. 10 (1997), 371-392. CMP 97:06

[4] M. Brion and M. Vergne, An equivariant Riemann-Roch theorem for complete, simplicial toric varieties, J. reine angew. Math. 482 (1997), 67-92. CMP 97:06

[5] M. Brion et M. Vergne, Une formule d'Euler-MacLaurin pour les fonctions de partition, C. R. Acad. Sci. Paris (Série I) 322 (1996), 217-220. MR 97a:11164

[6] M. Brion et M. Vergne, Une formule d'Euler-MacLaurin pour les polytopes convexes rationnels, C. R. Acad. Sci. Paris (Série I) 322 (1996), 317-320. MR 97a:11165

[7] S. E. Cappell and J. L. Shaneson, Genera of algebraic varieties and counting of lattice points, Bull. Amer. Math. Soc. 30 (1994), 62-69. MR 94f:14018

[8] S. E. Cappell and J. L. Shaneson, Euler-MacLaurin expansions for lattices above dimension one, C. R. Acad. Sci. Paris (Série I) 321 (1995), 885-890. MR 96i:52012

[9] R. Diaz and S. Robins, The Ehrhart polynomial of a lattice $n$-simplex, Electronic Research Announcements of the AMS 2 (1996). CMP 96:17

[10] E. Ehrhart, Polyèdres et réseaux, J. reine angew. Math. 226 (1967), 1-29. MR 35:4184

[11] V. Ginzburg, V. Guillemin and Y. Karshon, Cobordism techniques in symplectic geometry, The Carus Mathematical Monographs,, Mathematical Association of America, to appear.

[12] V. Guillemin, Riemann-Roch for toric orbifolds, preprint (1995).

[13] M-N. Ishida, Polyhedral Laurent series and Brion's equalities, International J. Math. 1 (1990), 251-265. MR 91m:14081

[14] L. C. Jeffrey and F. C. Kirwan, Localization for non-abelian group actions, Topology 34 (1995), 291-327. CMP 95:08

[15] J-M. Kantor and A. Khovanskii, Une application du théorème de Riemann-Roch combinatoire au polynôme d'Ehrhart des polytopes entiers de $\mathbb{R}^{d}$, C. R. Acad. Sci. Paris (Série I) 317 (1993), 501-507. MR 94k:52018

[16] T. Kawasaki, The Riemann-Roch theorem for complex $V$-manifolds, Osaka J. Math. 16 (1979), 151-159. MR 80f:58042

[17] A. Khovanskii and A. Pukhlikov, A Riemann-Roch theorem for integrals and sums of quasipolynomials over virtual polytopes, St-Petersburg Math. J. 4 (1993), 789-812. MR 94c:14044

[18] P. McMullen, Transforms, diagrams and representations, Proc. Geometry Symposium, Siegen 1978, Birkhäuser, Basel, 1979, pp. 92-130. MR 81i:52007

[19] R. Morelli, A Theory of Polyhedra, Adv. Math. 9 (1993), 1-73. MR 94f:52023

[20] B. Sturmfels, On vector partition functions, J. Combinatorial Theory, Series A 72 (1995), 302-309. MR 97b:52014

[21] M. Vergne, Equivariant index formulas for orbifolds, Duke Math. J. 82 (1996), 637-652. CMP 96:12

ABSTRACT. We obtain residue formulae for certain functions of several variables. As an application, we obtain closed formulae for vector partition functions and for their continuous analogs. They imply an Euler-MacLaurin summation formula for vector partition functions, and for rational convex polytopes as well: we express the sum of values of a polynomial function at all lattice points of a rational convex polytope in terms of the variation of the integral of the function over the deformed polytope.

Institut Fourier, B.P. 74, 38402 Saint-Martin D'Hères Cedex, France

E-mail address: mbrion@fourier.ujf-grenoble.fr

École Normale Supérieure, 45 rue D'Ulm, 75005 Paris Cedex 05, France

E-mail address: vergne@dmi.ens.fr 